\title{
Variability of the transitional T Tauri star T Chamaeleontis ${ }^{\star \star \star}$
}

\author{
E. Schisano ${ }^{1,2}$, E. Covino ${ }^{1}$, J. M. Alcalá ${ }^{1}$, M. Esposito ${ }^{3}$, D. Gandolfi ${ }^{4}$, and E. W. Guenther ${ }^{4}$ \\ 1 INAF - Osservatorio Astronomico di Capodimonte, via Moiariello 16, 80131 Napoli, Italy \\ e-mail: schisano@oacn.inaf.it \\ 2 Università degli Studi di Napoli "Federico II", Corso Umberto I, 80138 (NA) Italy \\ 3 Hamburger Sternwarte, Gojenbergsweg 122, 21029 Hamburg, Germany \\ 4 Thüringer Landessternwarte Tautenburg, Sternwarte 5, 07778 Tautenburg, Germany \\ Received 2 October 2008 / Accepted 9 March 2009
}

\section{ABSTRACT}

Context. It is known that for solar-mass stars planet formation begins in a circumstellar disc. The study of transitional objects exhibiting clear signs of evolution in their discs, such as the growth of dust particles and beginning of disc dispersal, is fundamental to understanding the processes governing dust-grain coagulation and the onset of planet formation.

Aims. We attempt to characterise the physical properties of T Chamaeleontis, a transitional T Tauri star showing UX Ori-type variability, and of its associated disc, and probe the possible effects of disc-clearing processes.

Methods. Different spectral diagnostics were examined, based on a rich collection of optical high- and low-resolution spectra. The cross-correlation technique was used to determine radial and projected rotational velocities, shape changes of photospheric lines were analysed via bisector-method applied to the cross-correlation profile, and the equivalent widths of both the Li I $\lambda 6708 \AA$ photospheric absorption and the most prominent emission lines (e.g., $\mathrm{H} \alpha, \mathrm{H} \beta$ and [OI] $6300 \AA$ ) were measured. Variability in the main emission features was inspected by means of line-profile correlation matrices. Available optical and near-infrared photometry combined with infrared data from public catalogues was used to construct the spectral energy distribution (SED) and infer basic stellar and disc properties.

Results. Remarkable variability on timescale of days in the main emission lines, $\mathrm{H} \alpha$ changing from pure emission to nearly photospheric absorption, is correlated with variations in visual extinction of over three magnitudes, while the photospheric absorption spectrum shows no major changes. The strength of emission in $\mathrm{H} \alpha$ and $\mathrm{H} \beta$ is highly variable and well correlated with that of the [OI] lines. The structure of the $\mathrm{H} \alpha$ line-profile also varies on a daily time-span, while the absence of continuum veiling suggests very low or no mass accretion. Variations of up to nearly $10 \mathrm{~km} \mathrm{~s}^{-1}$ in the radial velocity of the star are measured on analogous timescales, but with no apparent periodicity. SED modelling confirms the existence of a gap in the disc.

Conclusions. Variable circumstellar extinction is inferred to be responsible for the conspicuous variations observed in the stellar continuum flux and for concomitant changes in the emission features by contrast effect.

Clumpy structures, incorporating large dust grains and orbiting the star within a few tenths of AU, obscure episodically the star and, eventually, part of the inner circumstellar zone, while the bulk of the hydrogen-line emitting-zone and outer low-density wind region traced by the [OI] remain unaffected. In agreement with this scenario, the detected radial velocity changes are also explainable in terms of clumpy material transiting and partially obscuring the star.

Key words. stars: variables: general - stars: pre-main sequence - stars: late-type - stars: individual: T Cha stars: circumstellar matter - stars: planetary systems: protoplanetary discs

\section{Introduction}

T Tauri stars (TTS) are low-mass $\left(M \leq 2 M_{\odot}\right)$ pre-main sequence (PMS) stars (Appenzeller \& Mundt 1989). They are commonly classified into two sub-groups, the classical TTS (cTTSs), which are surrounded by an optically thick disc from which they accrete material, and the weak TTS (wTTSs), presumably representing the final stages of accretion and discclearing processes (Bertout et al. 2007). Hence, the cTTS-wTTS dichotomy is ascribed to different physical processes associated with the evolution of these young solar-type stars and their circumstellar environment. The equivalent width of the

\footnotetext{
* Based on observations obtained at the European Southern Observatory at La Silla, Chile in program 63.I-0045(A); 65.I0089(A); 66.C-0616(A); 67.C-0155(A); 67.C-0155(B); 68.C-0292(A); 69.C-0207(A); 70.C-0163(A); 073.C-0355(A); 074.A-9018(A); 075.C0399(A-F).

$\star \star$ Tables 1,2 and 4, and Figs. 21-32 are only available in electronic form via http://www . aanda. org
}

$\mathrm{H} \alpha$ emission is commonly used as an empirical criterion to distinguish between cTTS and wTTS (White \& Basri 2003). However, due to possible variability, no clean distinction can be made between the two subgroups based on the $\mathrm{H} \alpha$ emission alone.

Besides the traditional cTTS versus wTTS scheme, Herbst et al. (1994) introduced an additional class, the early-type T Tauri stars (eTTSs), from simple analysis of the photometric variability. ETTSs are earlier than $\mathrm{K} 0$ and have large-amplitude irregular variations (up to $\sim 3$ mag in $V$ ) with no sign of continuum veiling. Within this class, those authors also included some Herbig Ae/Be stars (HAEBEs) of the UX Ori type (Herbst et al. 1999). Studying this kind of variability along with stellar mass, Bertout (2000) concluded that T Tauri stars can display a similar phenomenology. Well-studied cases such as RY Lup (Gahm et al. 1989) and AA Tau (Bouvier et al. 2003) support this hypothesis.

How can all these observations be interpreted coherently in a scenario of star and circumstellar environment evolution is still 
questioned. Grinin (1988) modelled this kind of variability with dusty clumps in Keplerian orbits that temporarily obscure the central star. The rapid temporal evolution of such events indicates that the dust clouds usually appear in the disc close to the dust sublimation radius, probably in the inner rim region (Natta et al. 2001; Dullemond et al. 2003). This model implies a high inclination angle, while imaging and near-infrared interferometry of some of those stars are inconsistent with it (see the review by Millan-Gabet et al. 2007, and references therein). Another scenario was proposed by Vinković \& Jurkić (2007), in which a dusty outflow creates a halo in the inner-disc region, where clumps of dust occasionally intercept the line of sight and mask the stellar photosphere.

$\mathrm{T}$ Tauri discs evolve on a timescale of a few million years and the circumstellar raw material from which planets are constructed is affected different processes. In the inner part of the disc $(\$ 10 \mathrm{AU})$, the gas is accreted onto the star or expelled by bipolar jets, while the outer part of the disc may be dissipated by photoevaporation (Clarke et al. 2001; Alexander \& Armitage 2007). On the other hand, dust grains settle in the midplane, grow in size, and form planetesimals (Sicilia-Aguilar et al. 2007; Dullemond \& Dominik 2005). As the disc becomes progressively optically thin, solid particles migrate inward or outward, depending on their size, due to the combined action of different perturbing forces (photophoresis, gravity, radiation pressure, and gas drag), until they reach a stability distance from the star (Hermann et al. 2007). It remains unclear how planetesimals are formed in circumstellar discs and whether the hypothetical dusty clumps in highly variable TTS are related or not to the evolution of circumstellar dust until the formation of planetesimals. Detailed studies of the observed properties of objects with intermediate characteristics, in-between those of cTTS and wTTS, are expected to provide key constraints on star and planet formation scenarios.

Observations in the infrared (IR) with the Spitzer satellite have provided a wealth of information for interpretating the spectral energy distribution (SED) of young stars (see Lada et al. 2006; Cieza et al. 2007; Evans et al. 2008, and references therein), allowing the development of new classification schemes and scenarios of disc evolution (e.g., Cieza 2008; Merín et al. 2008). In particular, young stellar objects with no IR excess shortward of $10 \mu \mathrm{m}$, but with significant excess emission at wavelengths longer than $\sim 24 \mu \mathrm{m}$, are interpreted as being in an evolutionary phase of disc clearing and are hence classified as transitional objects (Furlan et al. 2006; Najita et al. 2007). Evidence for gaps in the disc of transitional objects have also been reported (Brown et al. 2007; Espaillat et al. 2008). In one of these, $\mathrm{LkCa} 15$, the most likely mechanism for gap-opening in the disc seems to be planet formation (Espaillat et al. 2008). Disc clearing may also be due to the presence of a companion, as in the case of CoKu Tau/4 (Ireland \& Kraus 2008).

Here we present a synoptic study of the spectroscopic variability of T Chamaeleontis (hereafter TCha), a transitional T Tauri star (Brown et al. 2007) displaying a UX Ori-like behaviour. TCha was observed in the course of a project aiming to reveal and monitor young spectroscopic binary systems (Esposito et al. 2006; Guenther et al. 2007).

The scheme of the paper is the following. In Sect. 2 we summarise the main observed properties of TCha as known from previous studies. In Sect. 3 we present the observations and data reduction, while in Sect. 4 we describe the radial velocity and $v \sin i$ determinations. In Sects. 5 and 6, we report the analysis performed on the spectrum of the object, focusing on the nonphotospheric contribution and, in particular, on the variability of the most prominent emission features (e.g., $\mathrm{H} \alpha, \mathrm{H} \beta$, and the [OI] $6300 \AA$ lines). In Sect. 8 we analyse the spectral energy distribution of TCha, while in Sect. 9 we discuss the results and present our interpretation of the object.

\section{Observed properties of T Cha}

T Cha shows strong photometric variability (up to 3 mag in the $V$-band) mainly characterised by erratic changes (Hoffmeister 1958; Mauder \& Sosna 1975; Covino et al. 1992), and by a UX Ori-like behaviour (i.e., the star becomes redder as it fades), with a tight correlation between brightness and colour (Alcalá et al. 1993; Covino et al. 1996). A periodicity of 3.2 was found by Mauder \& Sosna (1975). Spectroscopic variability in the most prominent emission lines was reported by Gregorio-Hetem et al. (1992) and Alcalá et al. (1993).

The PMS nature of T Cha was established by Alcalá et al. (1993) on the basis of the lithium criterion combined with the $\mathrm{H} \alpha$ emission strength and the presence of strong IR excess emission, while the spectral type G8 V (Alcalá et al. 1993) is earlier than that of a typical T Tauri star. From the strength of the $\mathrm{H} \alpha$ emission in former spectra, T Cha had been initially regarded as a wTTS (Alcalá et al. 1993), but later this classification turned out to be inconsistent with the strong variability of the star (Alcalá et al. 1995; Geers et al. 2006; Gras-Velazques \& Ray 2005). Moreover, besides the IR excess indicating the presence of circumstellar material, the object occasionally exhibits forbidden neutral oxygen lines that are not seen in wTTS spectra. Brown et al. (2007) modelled the SED and interpreted it in terms of a transitional disc.

TCha is located near the edge of the small cloud G300.2-16.9, also known as the Blue Cloud, in the direction of the Chamaeleon dark cloud complex (Nehme et al. 2008). In the Hipparcos Catalogue, a distance of $66_{-12}^{+19} \mathrm{pc}$ is reported, although the error in the parallax is probably far higher because of the strong variability of the star (reported to vary between 10.4 and $13.4 \mathrm{mag}$ in the $H_{P}$-band during the period of observation). More reliable estimates of the distance were obtained from proper motion studies by Frink et al. (1998) and by Terranegra et al. (1999), suggesting that T Cha is part of an association of stars at nearly $100 \mathrm{pc}$. Here we adopt the latter value.

\section{Observations and data reduction}

\subsection{High-resolution spectroscopy}

Data were acquired at the ESO-La Silla Observatory using the echelle spectrograph FEROS (Fiber-fed Extended Range Optical Spectrograph), first installed at the $1.5 \mathrm{~m}$ and, since October 2002 , then mounted at the $2.2 \mathrm{~m}$ telescope. The high resolving power $(R \sim 48000)$ and the wide useful spectral range (3600-9200 A) make FEROS particularly suitable for radialvelocity (RV) monitoring and spectral-line variability studies. FEROS was operated in the Object + Sky configuration, in which a sky spectrum is acquired simultaneously with the object through an adjacent fiber.

From 1999 to 2006, we obtained a total of 50 FEROS spectra with quite different signal-to-noise ratios $(S / N)$, due not only to the change of telescope or different observing conditions but also to the strong variability of the star. The spectra were acquired within the framework of a project on young spectroscopic binary systems. For that reason, the time coverage of our data is rather uneven, with some spectra acquired on daily temporal base and others separated by several months. 
The data reduction was performed using the specific FEROS Data Reduction Software (DRS) implemented in the ESOMIDAS $^{1}$ environment. The reduction steps were the following: bias subtraction and bad-column masking; definition of the echelle orders on flat-field frames; subtraction of the background diffuse light; order extraction; order by order flat-fielding; determination of wavelength-dispersion solution by means of ThAr calibration-lamp exposures; rebinning to a linear wavelengthscale $(\Delta \lambda=0.03 \AA)$ with barycentric correction; and merging of the echelle orders. All the spectra were then normalized to the continuum.

More details about the data reduction procedure and technical specifications of the instrument can be found at the FEROS Web site ${ }^{2}$. We emphasise that the high stability of the instrument allows an internal accuracy in the wavelength calibration of approximately $200 \mathrm{~m} \mathrm{~s}^{-1}$ (average residual $\mathrm{rms} \sim 3.5 \times 10^{-3} \AA$ ). A $\log$ of the FEROS observations is provided in Table 1.

Apart from the FEROS data, we also report measurements from a previous set of six high-resolution CASPEC spectra obtained simultaneously with optical broad-band photometry on two consecutive nights, 1994 January 31 and February 1 (Covino et al. 1996). Details about the instrumental set-up and data reduction can be found in Covino et al. (1997).

\subsection{Low-resolution spectroscopy}

In addition to the high-resolution spectroscopy, we include unpublished low-resolution $(R \sim 2000)$ spectra gathered during various observing runs conducted between 1993 and 1995 using the Boller \& Chivens spectrograph at the ESO $1.5 \mathrm{~m}$ telescope at La Silla, Chile. The reduction of these data was performed as described in Alcalá et al. (1995). We recall that these spectra were calibrated in relative fluxes using a standard star.

\section{Cross-correlation function analysis}

\subsection{Radial velocity determinations}

We used the cross-correlation technique to determine the radial velocity of TCha from the FEROS spectra. As a template, we chose a spectrum of the G8.5 V star HD 152391 $\left(\mathrm{RV}=44.8 \mathrm{~km} \mathrm{~s}^{-1}\right.$ and $v \sin i=3 \mathrm{~km} \mathrm{~s}^{-1}$ ) (Nordström et al. 2004), observed with the same instrumental set-up.

We followed the prescription by Esposito et al. (2006), i.e., a Gaussian was fitted to the peak of the cross-correlation function $(\mathrm{CCF})$, computed in six distinct spectral ranges. In some cases, the Gaussian fit failed, and in such cases we calculated the moments of the CCF profile and took the first-order moment as an $\mathrm{RV}$ estimator. In the end, we adopted as RV value and associated error, the average of the RV determined in the different ranges and their rms dispersion, respectively. All RV measurements are reported in Table 1 . The RV values vary with time ranging between 6 and $30 \mathrm{~km} \mathrm{~s}^{-1}$. A period search was performed on these data, but no clear indication of periodicity was found.

\subsection{CCF bisector analysis}

The Gaussian fit may sometimes appear inadequate due to asymmetries in the CCF peak. Asymmetries in stellar absorption lines can arise for several reasons and may be conveniently represented by line bisectors (Gray 2005). In some cases, variations in

\footnotetext{
${ }_{1}^{1}$ Munich Image Data Analysis System.

${ }^{2}$ http://www.ls.eso.org/lasilla/sciops/2p2/E2p2M/FEROS
}

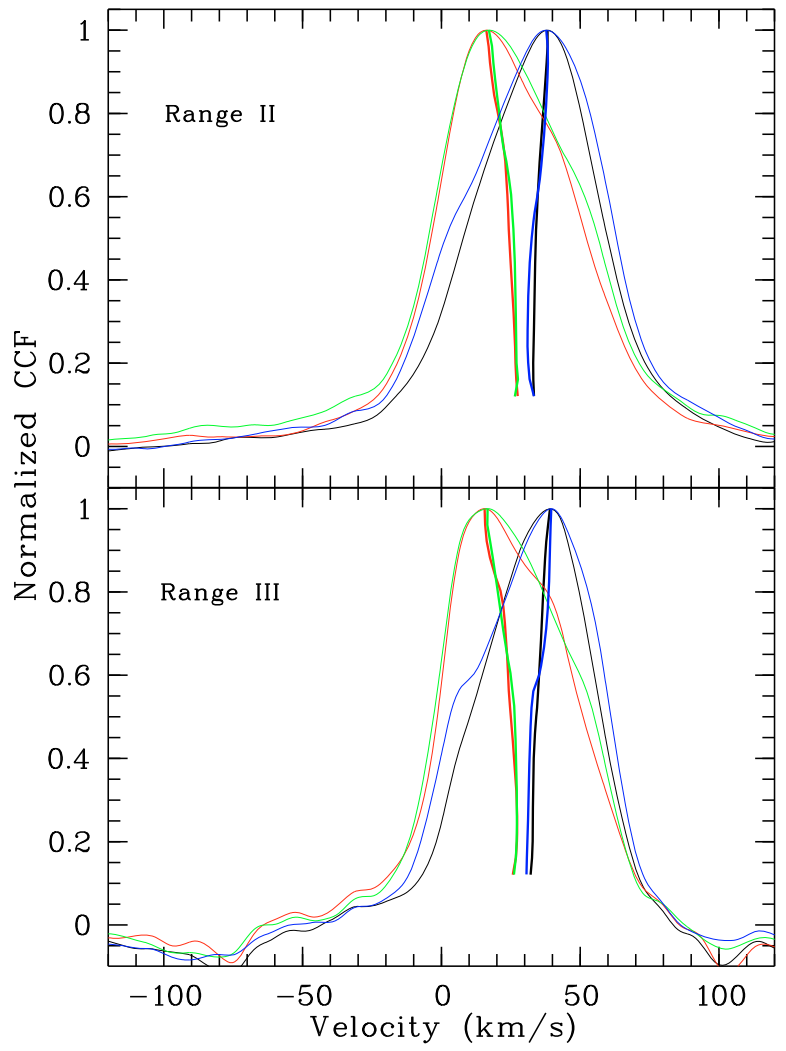

Fig. 1. Changes in the CCF in the period 19-23 May 2000, in two different wavelength ranges. The different colours refer to the following dates: 19 May (black), 21 May (red) 22 May, (green), 23 May (blue). The corresponding bisectors are also traced. Velocity is relative to the template star HD 152391.

the bisector shape may arise due to photospheric spots crossing the stellar disc as the star rotates, whereas uncorrelated variations in both bisector position and shape may indicate an unresolved spectroscopic system (Santos et al. 2002; Dall et al. 2006).

From the correlation between RV changes and bisector orientation, Queloz et al. (2001), in the case of HD 166435, stated that the periodicity in their RV data was not due to an unseen secondary object orbiting the star, but to stellar activity. We adopted the same tool to verify whether RV shifts measured by a Gaussian fit are purely due to changes in the CCF peak shape, or reflect true changes in the radial velocity of the star. An example of the variability of the CCF peak (and corresponding bisector) of T Cha on daily timescales is shown in Figs. 1 and 2. For each of the six spectral ranges considered in Sect. 4.1, we divided the corresponding CCF bisector into two intervals as in Queloz et al. (2001): an upper part, where the strongest changes occur, and a lower one, where the bisector position appears more stable (see in Fig. 2). The RV difference between the top and bottom bisector's mean velocities yields information about the bisector orientation. This quantity, called "bisector velocity span" (Toner \& Gray 1988; Queloz et al. 2001; Dall et al. 2006), is a good measure of the changes in bisector orientation, and hence of the asymmetry of the peak. The variations in the central part of the $\mathrm{CCF}$, which reflect the higher stability of the wings compared to the peak can be easily discerned.

The comparison between the RV values obtained from a Gaussian-fit and by a bisector analysis is shown in Fig. 3. The left panel shows how the RV values from a Gaussian-fit are well 


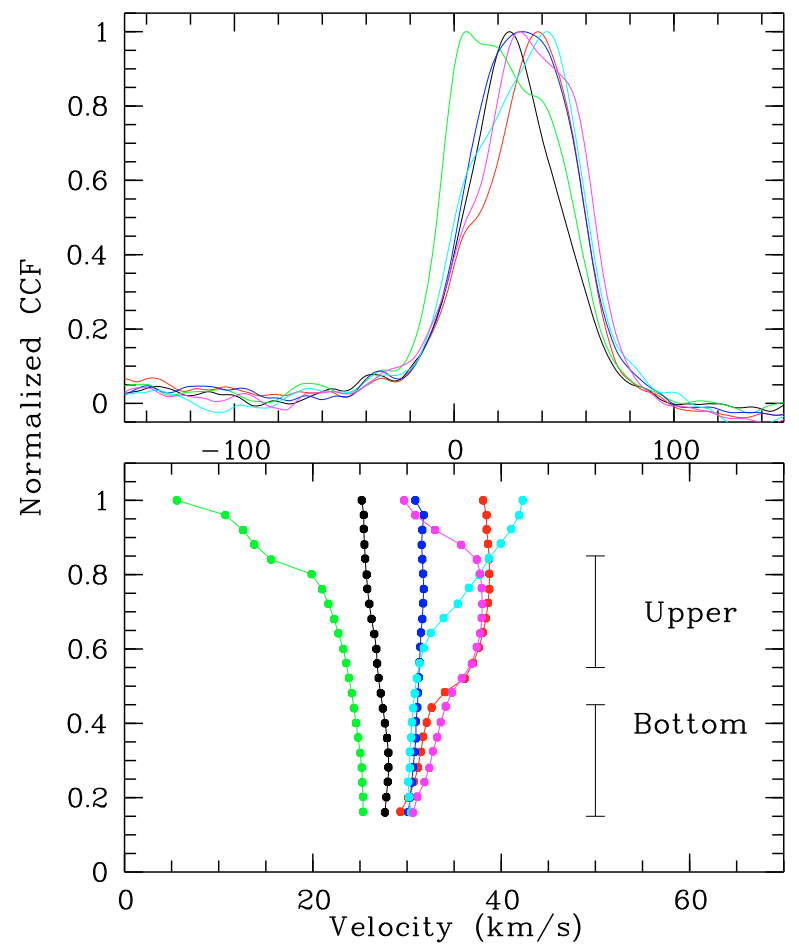

Fig. 2. CCF peaks between 16 and 22 May 1999 (upper panel). Velocity is relative to the template star. The different colours refer to the following dates: 16 May (black), 17 May (red), 18 May (green), 19 May (blue), 21 May (cyan), 22 May (pink). The lower panel shows, on expanded scale, the corresponding CCF bisectors.

correlated with the bisector velocity $\operatorname{span}^{3}$. The Gaussian function reproduces well only one of the two sides of an asymmetric CCF peak, leading to a systematic shift in the measured RV. On the other hand, the bisector values, at the bottom part of the profile, versus the bisector span show a weaker correlation than the Gaussian-fit values (Fig. 3 right panel).

The RV determinations derived from the bisector method, although of lower amplitude than the values derived from the Gaussian-fit method, confirm that RV is indeed variable, considering the average uncertainty of $1.7 \mathrm{~km} \mathrm{~s}^{-1}$. We performed Fourier analysis, using the formulation of the periodogram given by Scargle (1982), to search for possible periodicities in the radial velocity data, although no clear values were identified. This might in part be due to the extremely uneven temporal sampling. We had only one relatively long run of six consecutive nights, but when analysing this run alone, no hint of periodicity was found on a timescale of a few days.

Although the presence of blended spectroscopic components cannot be excluded, the lack of a clear periodicity prevents us from drawing firm conclusions about the possible binarity or multiple-nature of T Cha. As in Queloz et al. (2001), the correlations between the RV values from the Gaussian-fit method and the "velocity span" indicate as most plausible explanation the presence of inhomogeneities of variable extent moving across the photospheric disc, either intrinsic to the star (e.g., cool spots), or external to it (e.g., clustered orbiting material). Hence, in the following, T Cha will be treated as a single star. For the purposes of photospheric spectrum subtraction (see Sect. 5.2), we used the bottom bisector's mean RV value.

\footnotetext{
3 All RV measurements are reported in Table 2, available electronically.
}

\subsection{Projected rotational velocity}

We evaluated the projected rotational velocity, $v \sin i$, of T Cha by measuring the full width at half maximum (FWHM) of the $\mathrm{CCF}$ peak for the spectra with higher signal-to-noise ratio. We first established a relation between the FWHM and $v \sin i$, as described in Covino et al. (1997), using the template spectrum of HD 152391 broadened artificially by various $v \sin i$ values. The $v \sin i$ value determined in this way was $37 \pm 2 \mathrm{~km} \mathrm{~s}^{-1}$. The same measurement was repeated on an average spectrum obtained by combining a total of 34 spectra with $S / N \gtrsim 50$ around $6000 \AA$. The $v \sin i$ determined in the average spectrum is consistent with the previous value. However, when extending the measurements to all spectra, we identified a few with narrower spectral lines, yielding a $v \sin i$ of nearly $30 \mathrm{~km} \mathrm{~s}^{-1}$. Hence, we checked the $v \sin i$ determinations by directly fitting each spectrum with an artificially broadened template, which confirmed that some spectra had smaller line widths.

In Fig. 4, we report the $v \sin i$ values obtained from CCF Gaussian-fit FWHM calibration versus the width at half maximum of the CCF peak measured directly. In this case, it is obvious that the width of the lines does not provide a reliable measure of the rotational velocity. A variable line-width is indicative of either blended spectroscopic components or the presence of inhomogeneities moving across the stellar disc that alter the shape of photospheric lines. However, no correlation is found between the RV (either from a Gaussian-fit or bisector method) and the $v \sin i$ measurements.

From a periodogram analysis based on data points from the spectra of higher signal-to-noise ratio, we found a peak at about 0.25 cycles day $^{-1}$, or a period close to 4 days. This period does not differ dramatically from that found by Mauder \& Sosna (1975) and might reflect a rotational modulation induced by photospheric spots or other types of inhomogeneities transiting over the stellar disc.

\section{The spectrum of T Cha}

\subsection{Photospheric spectrum and lithium abundance}

The spectrum of T Cha is that of a G8 V star with strong absorption in the LiI resonance line at $\lambda 6708 \AA$ and a few emission lines typical of TTS. The spectral type does not change from one spectrum to the other, as also demonstrated in Sect. 7. Figure 5 shows the good match between the photospheric spectrum of T Cha and that of the template. The equivalent width $(E W)$ of the lithium line was measured in each spectrum of our FEROS dataset. The measurements were found to be internally consistent, within the errors, yielding a mean value of $E W_{\mathrm{Li}}=360 \pm 30 \mathrm{~m} \AA$. Using the calibration of Pavlenko \& Magazzú (1996) and adopting the temperature of $5000 \mathrm{~K}$, we derived a lithium abundance compatible with the cosmic value $\left(\log N_{\mathrm{Li}}=3.2\right)$, with an uncertainty of 0.2 dex. It is important to recall that this determination is strictly dependent on the effective temperature. At a temperature of $5500 \mathrm{~K}$, as adopted by Kenyon \& Hartmann (1995) for a G8 V star, the abundance would increase to 3.6 dex, implying some lithium enrichment with respect to the interstellar value.

\subsection{Photospheric spectrum subtraction}

One of the main goals of this work was to analyse the variability in the emission lines and hence probe the circumstellar environment of T Cha. For this purpose, it is useful to remove the photospheric contribution. We subtracted the photospheric-line 

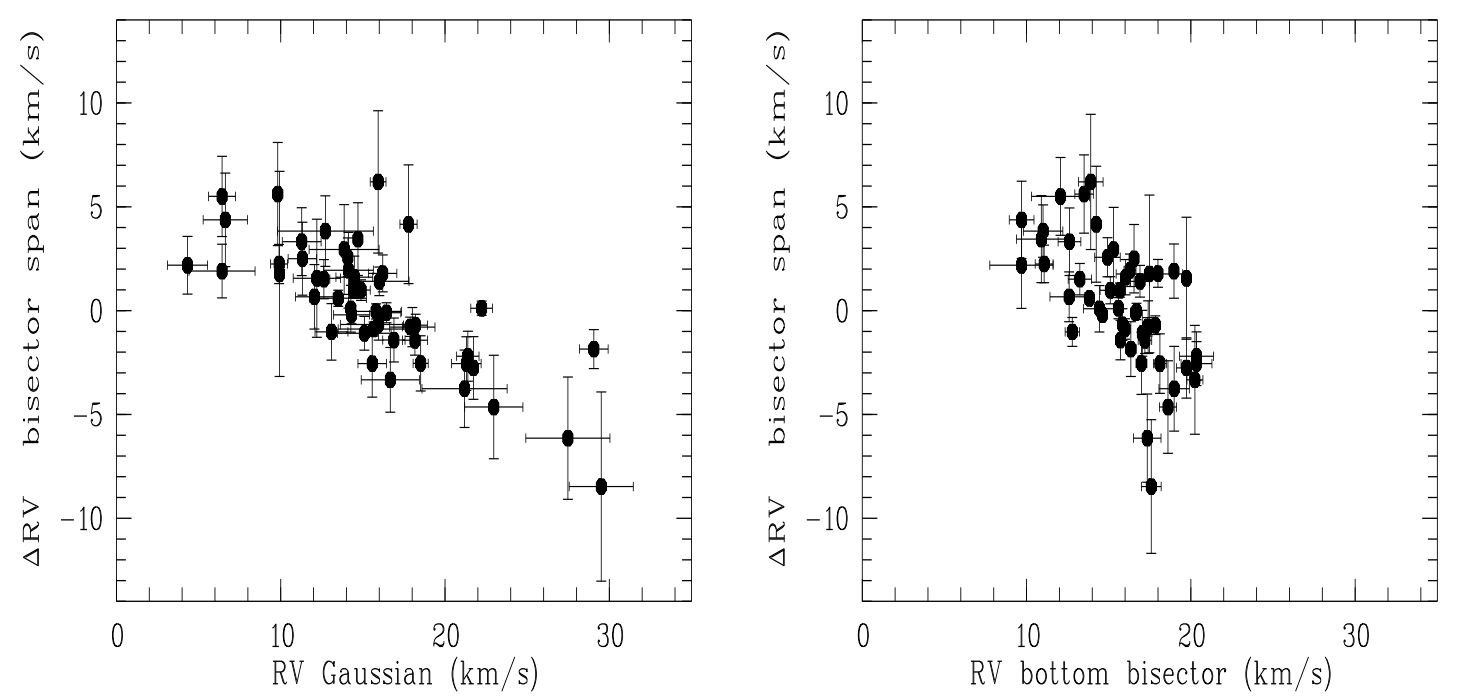

Fig. 3. The radial velocity measurements from Gaussian-fit method versus the "bisector velocity span" (i.e. the difference between the upper and bottom bisector mean velocities) are plotted in the left panel. The right panel shows, instead, the mean radial velocity of the CCF bisector bottom versus the "bisector velocity span".

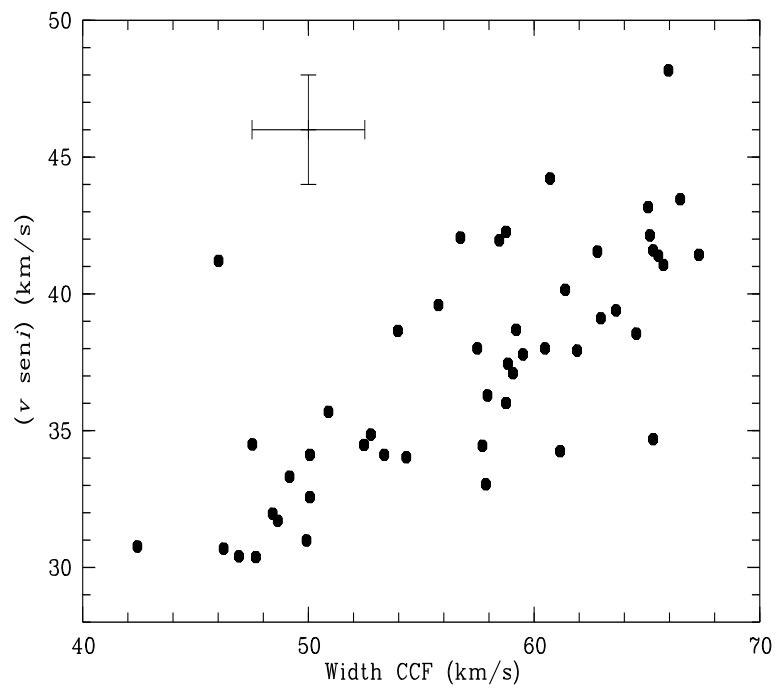

Fig. 4. Projected rotational velocity from CCF Gaussian-fit calibration versus FWHM of the CCF peak. Only the data of the highest $S / N$ are considered here.

spectrum from the FEROS spectra of T Cha using the same template spectrum of HD 152391 used in the cross-correlation analysis presented in Sect. 4.

All spectra were rebinned to the rest-wavelength frame to take account of their different RV shifts, derived from the $\mathrm{CCF}$ analysis described in Sect. 4.1. The template spectrum was broadened artificially by the $v \sin i$ value of $37 \mathrm{~km} \mathrm{~s}^{-1}$ and then subtracted from each of the TCha spectra. We assumed that the photospheric spectrum of a TTS resembles that of a mainsequence star of the same spectral type. The photospheric subtraction method also allows us to verify the possible presence of "veiling". No sign of continuum excess emission was found in T Cha (see Fig. 20), confirming the absence of UV-excess in the broad-band photometry (see Sect. 8 and Covino et al. 1996).
Examples of the residual profiles of the main emission lines at different dates are shown in Fig. 6. Some lines show absorption components shifted toward shorter and/or longer wavelengths. The $\mathrm{H} \alpha$ line is the most variable in both intensity and shape. The residuals of the NaI D lines show either redshifted or blue-shifted absorption components (that are easily distinguishable from the sharp, undisplaced, interstellar component), apparently linked to the presence of a correspondent feature in $\mathrm{H} \alpha$ and $\mathrm{H} \beta$. The sharp interstellar absorption components of the D lines from the associated cloud are also distinguishable, when not suppressed by the sky emission.

\subsection{Emission line spectrum}

Gregorio-Hetem et al. (1992), Alcalá et al. (1993) and Covino et al. (1996) already pointed out some outstanding properties of the variability in the emission lines, but here we exploit the wide spectral coverage of FEROS to analyse more lines simultaneously and identify the line profile changes at higher resolution than in previous works.

\subsubsection{The $\mathrm{H} \alpha$ line}

The most prominent emission line in the spectrum of T Cha is $\mathrm{H} \alpha$, which also exhibits impressive variability. The observed equivalent width of the line measured in the FEROS spectra ${ }^{4}$, ranges from about $0.3 \AA$ (on 14 Feb. 2002) to about $-30 \AA$ (on 8 Apr. 2002 $)^{5}$. Besides the variations in strength, from pure emission to absorption, the line profile is also highly variable in its structure. However, we do not observe any correlation between the profile of the line and its $E W$. The line extends approximately from -300 to $+300 \mathrm{~km} \mathrm{~s}^{-1}$. However, much of the emission is concentrated in an interval of almost $400 \mathrm{~km} \mathrm{~s}^{-1}$ in width centred on zero velocity. In most of the spectra, the $\mathrm{H} \alpha$ line exhibits both blue-shifted and redshifted absorption components superimposed on a nearly centred symmetric broad emission.

\footnotetext{
${ }^{4}$ Reported in Table 2, only available in electronic form.

5 Conventionally, a negative $E W$ indicates line in emission.
} 

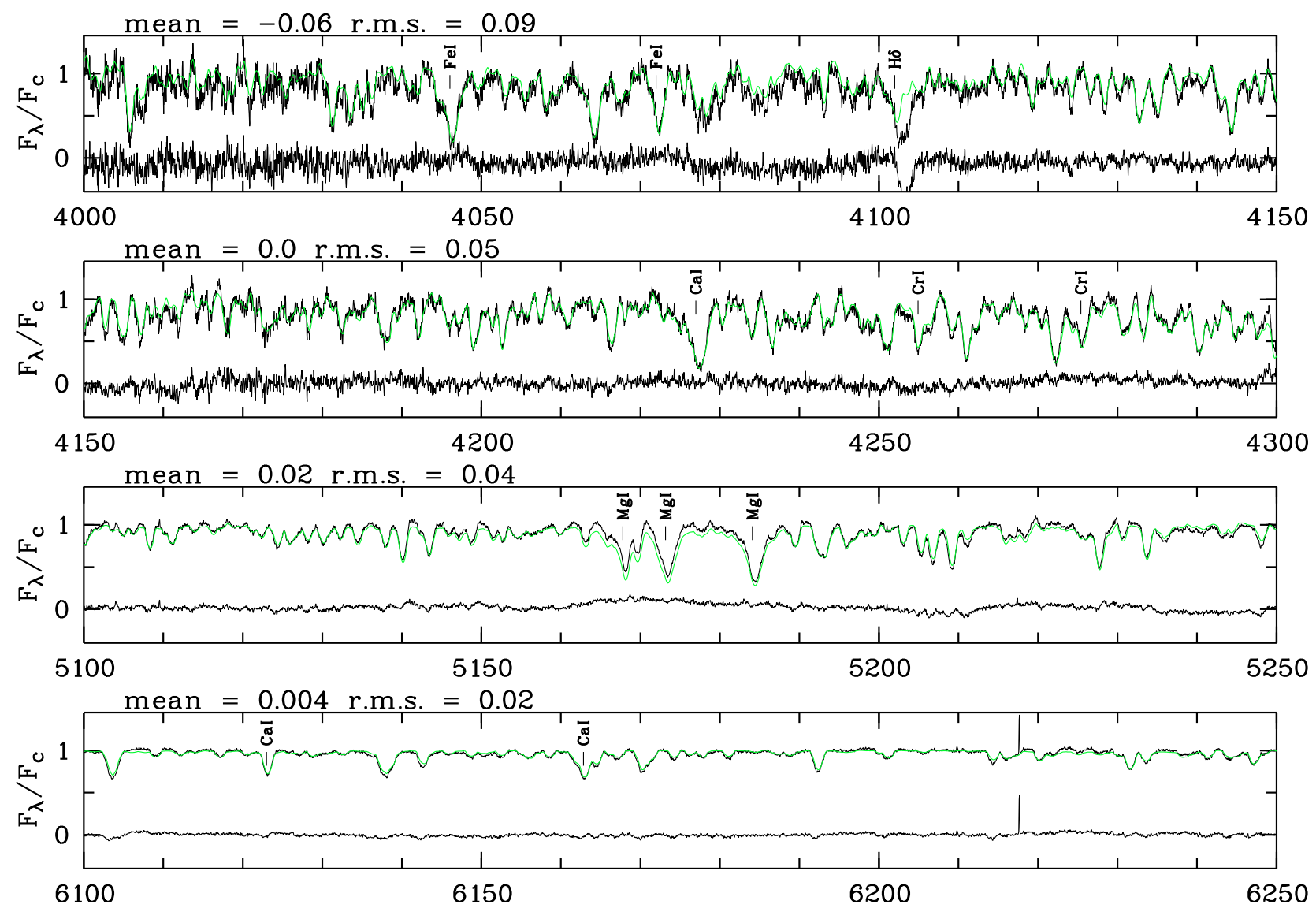

Fig. 5. Spectrum of T Cha obtained on 14 Feb. 2002. The green line represents the spectrum of the template (HD 152391 G8.5V) artificially broadened for rotation. In each panel is also shown the residual spectrum after subtraction. Slight residuals in strong spectral features are mainly due to uncertainties in the normalization to the continuum. The spectrum, with a good $S / N$, shows a redshifted absorption component at $\mathrm{H} \delta$.

The blue absorption is usually weaker than the red one, although in three spectra of January 2001 it appears stronger (e.g., date 51918 in Fig. 6). During five different epochs, the blue absorption reached below the continuum producing a PCygni profile. In most cases, when the emission component was not so strong, the red absorption was below the continuum, producing a typical inverse-P Cygni profile.

The red absorption is almost always present but with different velocity displacements relative to the rest wavelength, whereas the blue absorption sometimes becomes barely visible or disappears completely. In contrast, the emission on the blue side is generally more intense than the red one. This suggests that the red wing of the line is affected by significant absorption that causes the emission to appear weaker than in the blue wing. No clear regularity appears to exist in the line changes, but some profiles reappear after some time.

The lack of both detectable veiling and UV excess indicates that the accretion rate is rather low. Assuming that the $\mathrm{H} \alpha$ emission is entirely due to accretion, we estimated the mass accretion rate, $\dot{M}_{\text {accr }}$, by adopting the relationship found by Natta et al. (2004) between $\dot{M}_{\text {accr }}$ and the width of the $\mathrm{H} \alpha$ line at $10 \%$ intensity. We emphasise that this quantity does not represent an accurate mass-accretion estimator for two main reasons: i) it is not easy to measure, mainly due to the difficulty in defining the continuum level; and ii) because the relationship, derived for the substellar mass regime, shows a larger dispersion at higher masses. We determined the width at $10 \%$ intensity of the $\mathrm{H} \alpha$ emission only in spectra for which we could perform a
Gaussian decomposition of the line profile into an emission component with overlapping absorptions. From those, we derived a mean value of $\dot{M}_{\text {accr }}=4 \times 10^{-9} M_{\odot} \mathrm{yr}^{-1}$.

\subsubsection{The $\mathrm{H} \beta$ line and higher Balmer lines}

The strength of $\mathrm{H} \beta$ is also variable and its EW correlates well with that of $\mathrm{H} \alpha$, as shown in Fig. 7. The range of variation is between about $1.2 \AA$ (on 14 Feb. 2002) and about $-6 \AA$ (on 8 Apr. 2002). In most cases, the $\mathrm{H} \beta$ line presents typical doublepeak emission with higher emission in the blue peak than in the red one. Apart from when emission is stronger, the $\mathrm{H} \beta$ profile resembles the $\mathrm{H} \alpha$ one. However, the inverse P Cygni profile appears more frequently than in $\mathrm{H} \alpha$.

Even if $\mathrm{H} \alpha$ and $\mathrm{H} \beta$ have different optical thicknesses and formation regions, their $E W \mathrm{~s}$ are strongly correlated with each other (Fig. 7 lower panel).

Because of the lower efficiency of FEROS at shorter wavelengths and the high variability of T Cha in the $U$ and $B$ bands, little can be said about the behaviour of the higher members of the Balmer series, since $\mathrm{H} \gamma$ and $\mathrm{H} \delta$ are discernible only in a few spectra. Almost no emission is apparent in the residual spectrum, except in one or two cases, but the low $S / N$ still prevents us from drawing any robust conclusion. The most intense absorptions, either blue- or redshifted, depending on epoch, can also be identified in the higher Balmer lines (cf. $\mathrm{H} \delta$ in Fig. 5). 


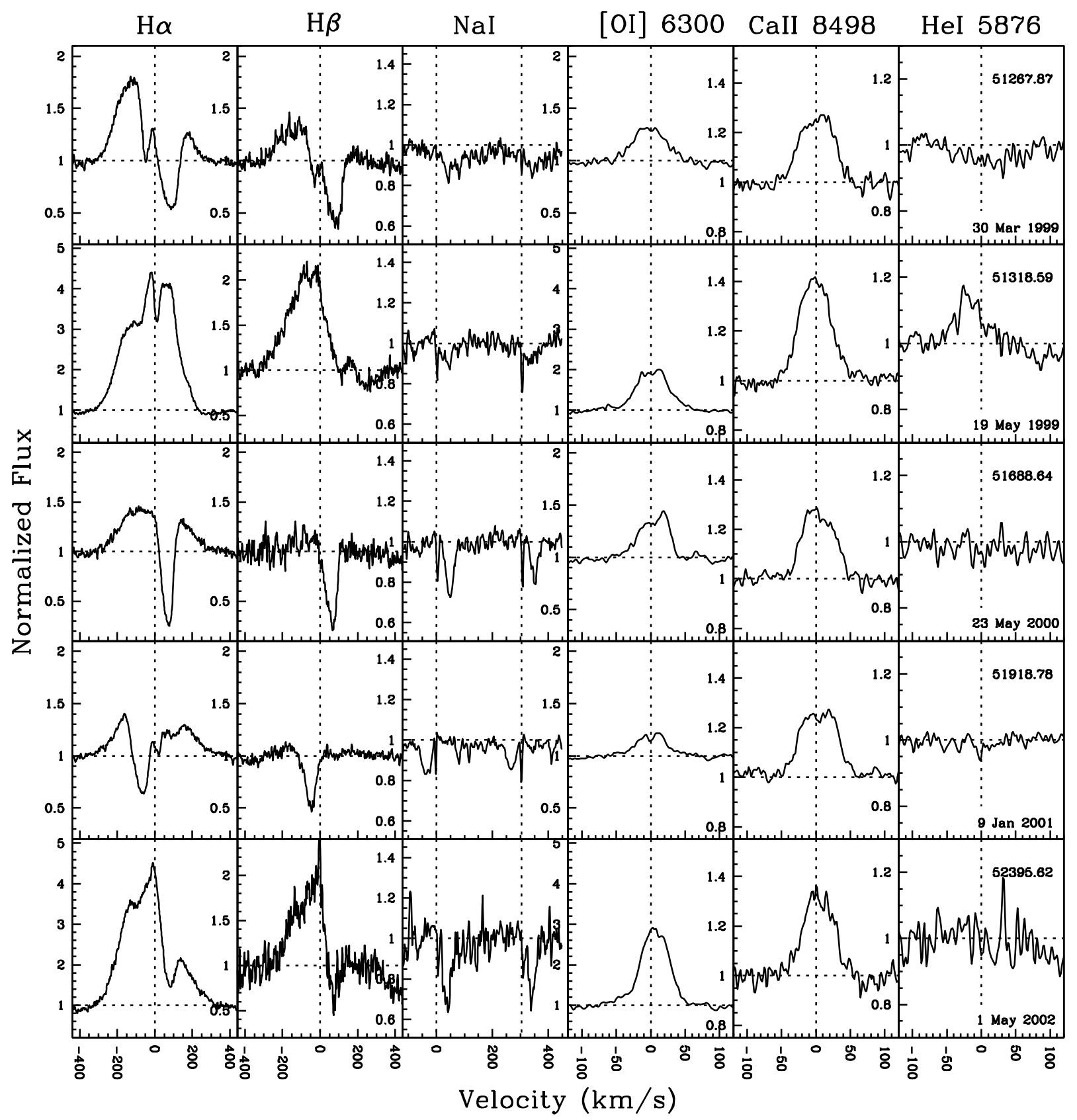

Fig. 6. Examples of residual line profiles of T Cha in velocity scale. The vertical dotted line marks the rest position in the star frame.

\subsubsection{Forbidden oxygen lines}

The forbidden [OI] 6300 and $6363 \AA$ emission lines are observed in the spectrum of T Cha. The contribution of the sky emission lines to the FEROS spectra was subtracted after scaling the sky spectrum by a factor to account for the different efficiencies of the object and sky fibers, determined from the ratio of the flatfield intensities in the corresponding fibers. Figure 6 shows the sky-subtracted line at $6300 \AA$. The width of $\sim 100 \mathrm{~km} \mathrm{~s}^{-1}$ places the line just on the edge between low- and high-velocity components for T Tauri stars (Hartigan et al. 1995).

As in the case of the Balmer lines, the forbidden oxygen lines are of variable intensity, but their profiles remain about the same. The most frequently observed profile is a symmetrical and well-centred emission, but sometimes the line is slightly redshifted by about $10-20 \mathrm{~km} \mathrm{~s}^{-1}$ (see Fig. 6).
The symmetrical profile suggests that both the approaching and receding parts of the flow contribute to the observed line, i.e., the star-disc system is viewed at relatively high inclination. Redshifted [OI] $6300 \AA$ profiles have also been observed in BP Tau and RW Aur (Hartigan et al. 1995), but are rather unusual for T Tauri stars. Some authors explain the redshifted component in terms of asymmetric outflows (e.g., Hirth et al. 1994; Hartigan et al. 1995).

Remarkably, the equivalent width of the [OI] $6300 \AA$ is correlated with that of the $\mathrm{H} \alpha$ emission (see Fig. 7 upper panel). As discussed in the following sections, this correlation indicates that the variations must be related to changes in the intensity of the underlying stellar continuum due to variable circumstellar extinction.

To verify the hypothesis of variable obscuration, we used the only simultaneous spectroscopy and photometry data 


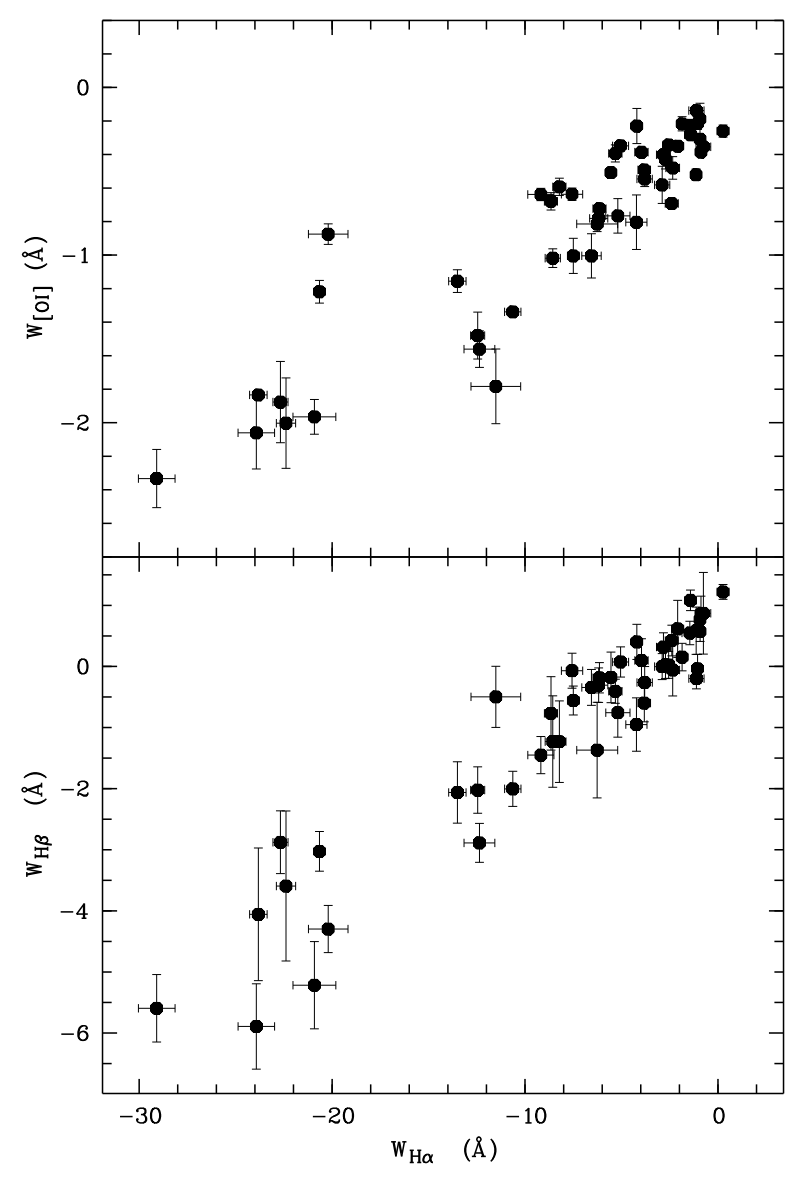

Fig. 7. Correlations between intensities of the most prominent emission lines measured in the FEROS spectra.

Table 3. Equivalent widths of [OI] $6300 \AA$ measured in CASPEC spectra (Covino et al. 1996) and line luminosity derived using simultaneous $R$-band photometry.

\begin{tabular}{cccc}
\hline \hline $\begin{array}{c}\text { JD } \\
-2440000\end{array}$ & $\begin{array}{c}R \\
\mathrm{mag}\end{array}$ & $\begin{array}{c}W_{\text {[OI] }} \\
(\AA)\end{array}$ & $\begin{array}{c}L_{\text {[OI] }} \\
\left(10^{28} \mathrm{erg} \mathrm{s}^{-1}\right)\end{array}$ \\
\hline 9384.62 & 11.47 & $-0.78 \pm 0.06$ & $5.4 \pm 0.4$ \\
9384.70 & 11.70 & $-0.91 \pm 0.08$ & $5.1 \pm 0.4$ \\
9384.87 & 11.88 & $-1.24 \pm 0.16$ & $5.9 \pm 0.8$ \\
9385.62 & 10.56 & $-0.32 \pm 0.03$ & $5.1 \pm 0.5$ \\
9385.76 & 10.41 & $-0.25 \pm 0.03$ & $4.6 \pm 0.5$ \\
9385.87 & 10.39 & $-0.24 \pm 0.03$ & $4.5 \pm 0.6$ \\
\hline
\end{tabular}

available to us (Covino et al. 1996) to evaluate the luminosity in the $[\mathrm{OI}]$ line,

$$
\begin{aligned}
L_{[\mathrm{O} \text { I }]} & =W_{[\mathrm{OI}]} \cdot f_{R} \cdot 4 \pi d^{2} \\
& =W_{[\mathrm{OI}]} \cdot 10^{-0.4 \cdot R} \cdot 2.69 \times 10^{33} \mathrm{erg} \mathrm{s}^{-1},
\end{aligned}
$$

where $W_{[\mathrm{OI}]}$ is the measured equivalent width of [OI], $d$ is the distance of $100 \mathrm{pc}$, and $f_{R}$ is the observed flux of the star in the $R$-band. The latter is given by $f_{R}=F_{0}^{R} \cdot 10^{-0.4 \cdot R}$, where $F_{0}^{R}=2.26 \times 10^{-9} \mathrm{erg} \mathrm{s}^{-1} \mathrm{~cm}^{-2} \AA^{-1}$ is the R-band flux of a zeromagnitude star (Bessel 1979). The measured EWs and relative luminosities are reported in Table 3.

The luminosity (or the flux) in the [OI] line remains relatively constant with time, as in the case of the UXOR star RR Tauri (Rodgers et al. 2002). Therefore, the strong variability in the emission-line intensity is caused by changes in the stellar continuum flux level rather than the intrinsic variability of the line-emitting region. This explains the correlation between $\mathrm{H} \alpha$ and [OI] discussed in Sect. 5.3.3, justifying the fact that the intensities of the two lines, originating in distinct regions, respond simultaneously to the brightness variations of the star. As the analysis of flux-calibrated low-resolution spectra presented in Sect. 7 shows, the intensities of the $\mathrm{H} \alpha$ and [OI] lines are also found to be related directly to the amount of circumstellar extinction affecting the photospheric continuum.

\subsubsection{Other emission lines}

Franchini et al. (1992) detected a weak variable emission component in the core of the NaI D lines. In our spectral subtraction analysis, we found no sign of this emission, but instead, were able to identify strong redshifted absorption in many residual spectra that is visible only when the redshifted absorption in $\mathrm{H} \alpha$ is also present (although the reverse is untrue), independently of the equivalent width of the $\mathrm{H} \alpha$ line. On three dates, namely 9 Jan. 2001, 21 Apr. 2002, and 9 Apr. 2005, when the blue-shifted absorption component in $\mathrm{H} \alpha$ was at least as strong as the red one, the feature appeared blue-shifted, but there was no sign of absorption on 14 Jan. 2001, when $\mathrm{H} \alpha$ showed a clear P Cygni profile.

The [NII] $6583 \AA$ forbidden emission line is occasionally observed in the low-resolution spectra when the star reaches its faintest state (see Sect. 7).

The He I emission line at $5876 \AA$ was seen on only two dates (19 May 1999 and 17 Feb. 2002), while missing in other spectra close to those dates. This line is probably caused by episodic, short-lived flare activity, common in young solar-type stars. As shown in Fig. 6, the line appears blue-shifted by about $-30 \mathrm{~km} \mathrm{~s}^{-1}$.

Finally, the emission cores of the two lines 8498 and $8662 \AA$ of the CaII IR triplet are clearly visible. The other line, at $8541 \AA$, falls in a wavelength gap in-between the echelle orders of FEROS. The equivalent widths of the two measurable lines remain almost constant, with small variations presumably related to chromospheric activity. No correlation is found with other emission lines. The profiles appear always symmetrical and well centred on the star velocity, indicating presumably a chromospheric origin, typical of young stars.

As for higher Balmer lines, the low $S / N$ prevented us from analysing the CaII $\mathrm{H}$ and $\mathrm{K}$ lines, but in some spectra of sufficiently high $S / N$, a strong emission core, indicative of intense chromospheric activity, is observed.

\section{Line profile variability on short timescale}

The large variety of $\mathrm{H} \alpha$ profiles shown by T Cha appear to occur rather erratically, although this may be caused at least partly by the quite uneven temporal sampling of our spectra.

We used three different series of spectra acquired with a time sampling shorter than one day to investigate variations on short timescales. We analysed separately each of the following three runs: 1) from 27 March 1999 to 1 April 1999 (total of 16 spectra in 5 nights); 2) from 16 to 22 May 1999 (total of 6 spectra in 6 nights); and 3) from 9 to 23 May 2000 (total of 5 spectra in 5 nights). Figure 8 shows in each panel the overlap of $\mathrm{H} \alpha$ (top-left box) and $\mathrm{H} \beta$ (bottom-left box) line profiles for each of the three runs. The average normalized profile $\left\langle I_{n}(\lambda)\right\rangle$ is also superposed, and is represented by a thicker line. The profiles, and their behaviour, appear to differ in each run, being more erratic in the second period than in the other two. The $\mathrm{H} \alpha$ and $\mathrm{H} \beta$ line 

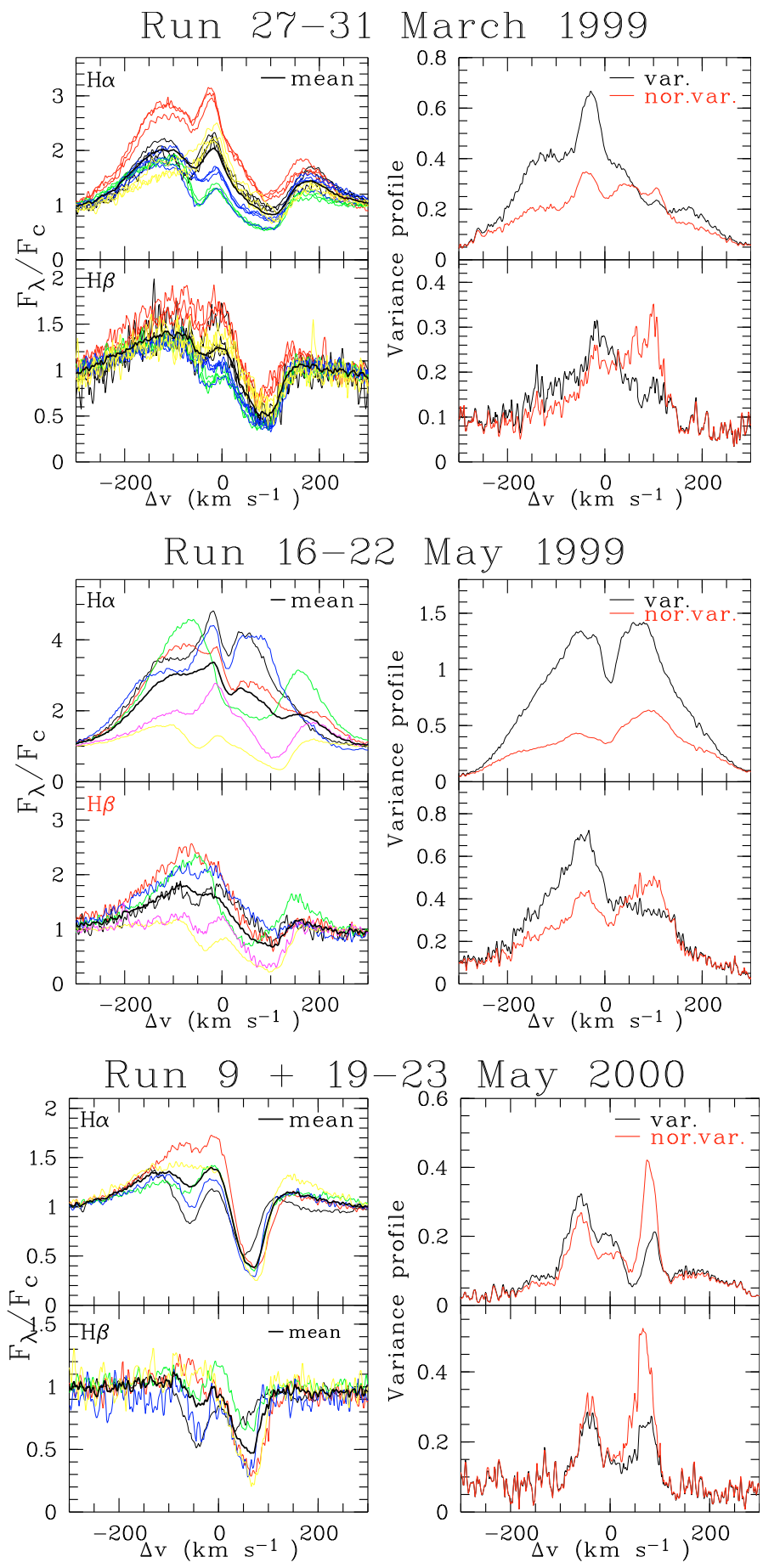

Fig. 8. Line profiles (left panels) and corresponding variance and normalized variance profiles (right panels) for $\mathrm{H} \alpha$ and $\mathrm{H} \beta$ in three different runs.

intensities and profiles change on a daily timescale, with only minor changes occurring during the night. We report in Fig. 10, the variability in the positions of the central peak and the two absorption components in $\mathrm{H} \alpha$ during the first run.

To quantify the variability in each line, we examined the variance, $\sigma$, and the normalized variance, $\sigma_{n}$, in the different runs, defined respectively as:

$\sigma^{2}=\Sigma_{i=1}^{N}\left[I_{n, i}(\lambda)-\left\langle I_{n}(\lambda)\right\rangle\right]^{2} /(N-1)$

and

$\sigma_{n}^{2}(\lambda)=\sigma^{2}(\lambda) /\left\langle I_{n}(\lambda)\right\rangle$
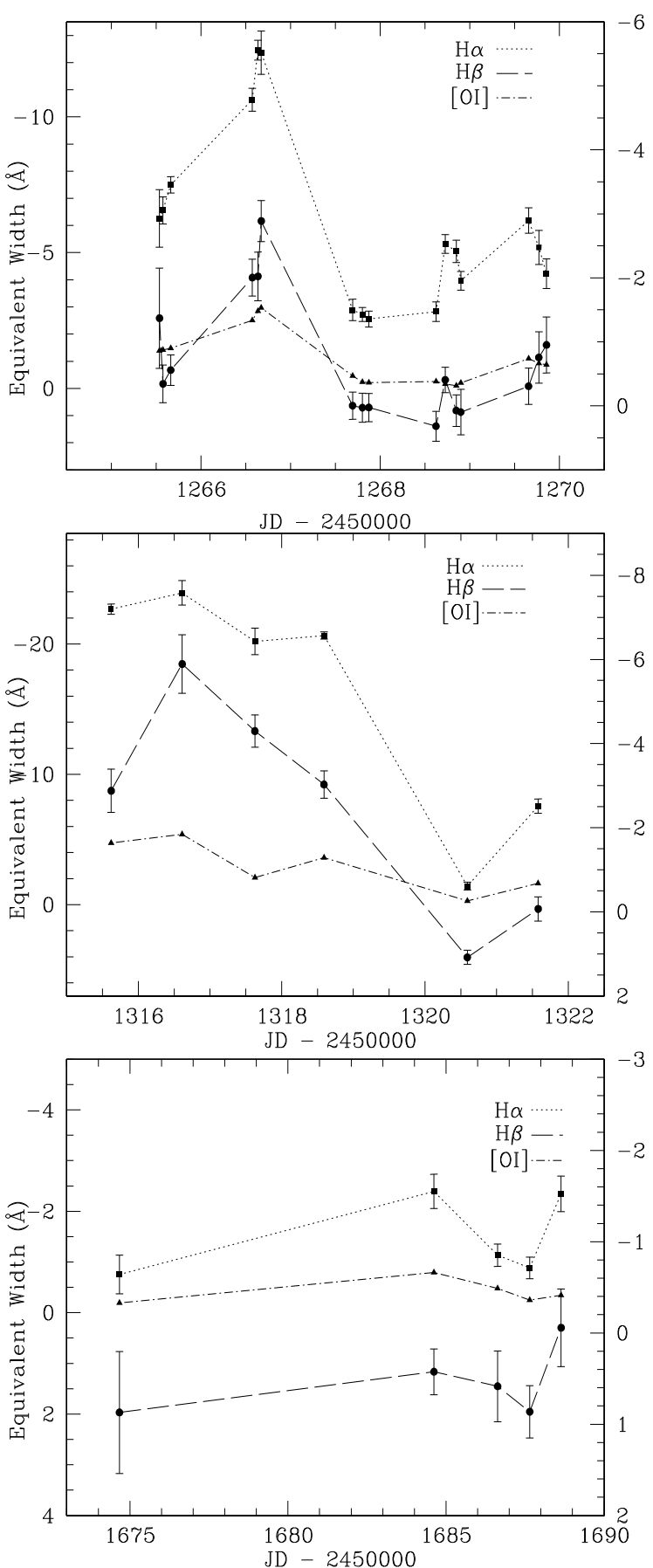

Fig. 9. Equivalent widths of the main emission lines during the runs displayed in Fig. 8. The left scale refers to $\mathrm{H} \alpha$, while the right one is for $\mathrm{H} \beta$ and [OI] lines.

where $I_{n, i}(\lambda)$ is the $i$ th spectrum. These quantities are plotted in the right panels of Fig. 8. If variability were merely caused by the brightening or fading of the stellar continuum flux, the shape of normalized variance profiles would be the same as the average line profile (Johns \& Basri 1995). This is generally not the case, suggesting that some other changes in the $\mathrm{H} \alpha$ forming region also occur.

Figure 9 shows the trends of the equivalent widths of the main emission lines with time. $\mathrm{H} \alpha$ and $\mathrm{H} \beta$ mainly vary in similar ways. On two dates, their behaviour is different, i.e., the last day of the first run, when the $\mathrm{H} \alpha$ intensity increased as the $\mathrm{H} \beta$ 


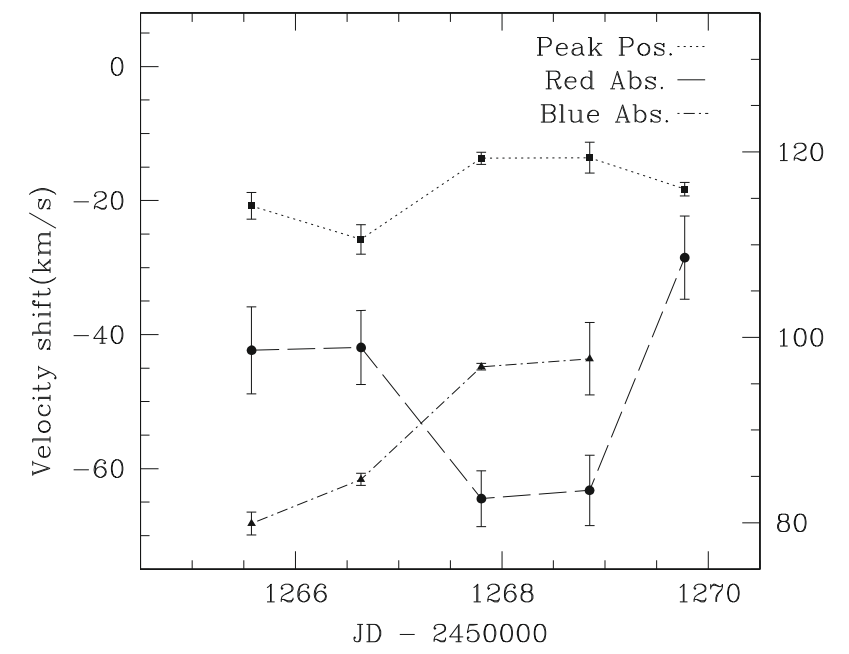

Fig. 10. Variations in position of the central peak emission and the two absorptions of $\mathrm{H} \alpha$ line during the period from 27 to 30 March 1999. The scale on the right refers to the red absorption, while the one on left refers to the central emission and the blue-shifted absorption.

intensity decreased, and the third day of the second run, when an increase in $\mathrm{H} \alpha$ intensity did not correspond to an increase in that of $\mathrm{H} \beta$. The [OI] line trend is similar to that of $\mathrm{H} \alpha$ except for during the fourth night of the first run and the last night of the third run, when the emission intensities of both Balmer lines strengthened, while the [OI] lines did not change. The similarities in the variability of these lines indicate that the processes producing the observed changes must affect contemporaneously circumstellar zones located at different distances from the star, i.e., on length scales of between a few tenths of $\mathrm{AU}$ for $\mathrm{H} \alpha$ and a few AUs for [OI].

\subsection{Correlation matrices}

To test whether the variations across the emission line profiles have a common origin, we computed their correlation matrices (CMs). CMs indicate the linear correlation coefficient between the variation in each velocity bin of the spectral line profile and variations in all other bins for the same or for two different lines. A strong correlation between different velocity bins is indicative of a common origin, or emitting region. If the variability in the emission line is linked to variability in the continuum, then the line profile is highly correlated over a wide range of velocity, producing a typical squarish shape in the CM (Johns \& Basri 1995; Alencar \& Batalha 2002).

We computed the CMs of $\mathrm{H} \alpha$ and $\mathrm{H} \beta$ with respect to themselves and to each other. Two-dimensional plots of these matrices for $\mathrm{H} \alpha$ and $\mathrm{H} \beta$ in three different runs are shown in Fig. 11. No anticorrelation is evident in the matrices. In each run, there are regions that correlate well with themselves, but do not correlate with the remainder of the line. No significant correlation is seen between the red $\left(\geq 200 \mathrm{~km} \mathrm{~s}^{-1}\right)$ and the blue $\left(\leq-200 \mathrm{~km} \mathrm{~s}^{-1}\right)$ wings of the profile. However, in the second run, the $\mathrm{H} \alpha$ line exhibits a correlation over a wide velocity range, its squarish form extending from about -300 to nearly $150 \mathrm{~km} \mathrm{~s}^{-1}$. The correlation is disrupted by the blue-shifted absorption, from -150 to $-50 \mathrm{~km} \mathrm{~s}^{-1}$, and the redshifted component, up to about $-100 \mathrm{~km} \mathrm{~s}^{-1}$, which do not correlate with the remainder of the line profile but with the redshifted emission peak, around $130 \mathrm{~km} \mathrm{~s}^{-1}$. This behaviour is not observed in the other epochs. Similarly, in the first run, the broad red absorption from -20 to $150 \mathrm{~km} \mathrm{~s}^{-1}$ breaks the correlation with the blue emission wing, which still correlates weakly with the red emission wing. Interestingly, the square regions in the CMs correspond to some of the highest peaks in the variance profiles, i.e., the blue emission wing, the central peak and the red absorption, respectively.

The $\mathrm{H} \beta \mathrm{CMs}$ are more affected by noise due to lower $S / N$ at those wavelengths, but again traces of a squarish form can be seen in particular in data from the second run. We also computed the $\mathrm{CM}$ between $\mathrm{H} \alpha$ and $\mathrm{H} \beta$, but, although the profiles of the two lines appear to be similar, the corresponding correlation matrices are affected too significantly by noise and no useful information can be extracted from them.

In conclusion, coherent changes in the velocity interval between -300 and $120 \mathrm{~km} \mathrm{~s}^{-1}$ appear consistent with variations dominated by variable circumstellar obscuration of the stellar photosphere, giving rise, due to a contrast effect, to changes in the relative intensity of emission lines. In the case of magnetospheric accretion, parts of the $\mathrm{H} \alpha$ profile, i.e., the redshifted component, are expected to originate in regions close to the star and eventually to be affected by direct obscuration. These may be identified kinematically with the intervals where the correlation breaks down whereas the differences from one epoch to the other possibly reflect changes in the geometry of the emitting region.

\section{Variability in the low-resolution spectra}

Strong variability was also detected in our low-resolution spectroscopy. We refer in particular to Table $4^{6}$, where we report the equivalent widths of the $\mathrm{H} \alpha$ and [OI] $\lambda 6300 \AA$ lines, and to Fig. 12, where a sample of low-resolution spectra is shown ${ }^{7}$.

We note that during the May-June 1993 run, the star exhibited spectacular changes between one night and the next. For instance, $\mathrm{H} \alpha$ was observed to vary between weak absorption $(E W \approx 0)$ on May 31 , to a very strong emission line $(E W \approx-50 \AA)$ on June 1 . The latter spectrum was also characterised by strong [OI] lines, and the [NII] line was also present, while no emission lines were detected in data acquired the two nights before.

The spectacular variability of these lines is illustrated in Fig. 13, where two low-resolution spectra, corresponding to close to the maximum and minimum brightness of the star, just two nights apart, are compared after normalising each of them to the flux at $\lambda=5880 \AA$. We recall that these spectra are calibrated in relative flux (see Sect. 3.2). The variation in the continuum slope between the two dates is also remarkable, in addition to the emission features appearing prominent when the photospheric continuum looks fainter and heavily reddened.

However, no change in the spectral type of the star is observed. To verify this, we used low-resolution spectra calibrated in relative flux, and applied the methods described in Alcalá et al. (2006) and Gandolfi et al. (2008) to determine simultaneously the spectral type and the visual extinction at the phases of minimum and maximum brightness, respectively. The result, shown in Fig. 14, is that the spectral type did not vary to within about half a sub-class, while the visual extinction changed from $1.2 \mathrm{mag}$ in the bright phase to about $4.6 \mathrm{mag}$ in the faint state, respectively. This corresponds to an extinction increase of at least 3.4 mag between the bright and faint states. By instead using the

\footnotetext{
${ }^{6}$ Available only in electronic form.

7 The full atlas of low-resolution spectra is reported in Fig. 21, only in electronic form.
} 

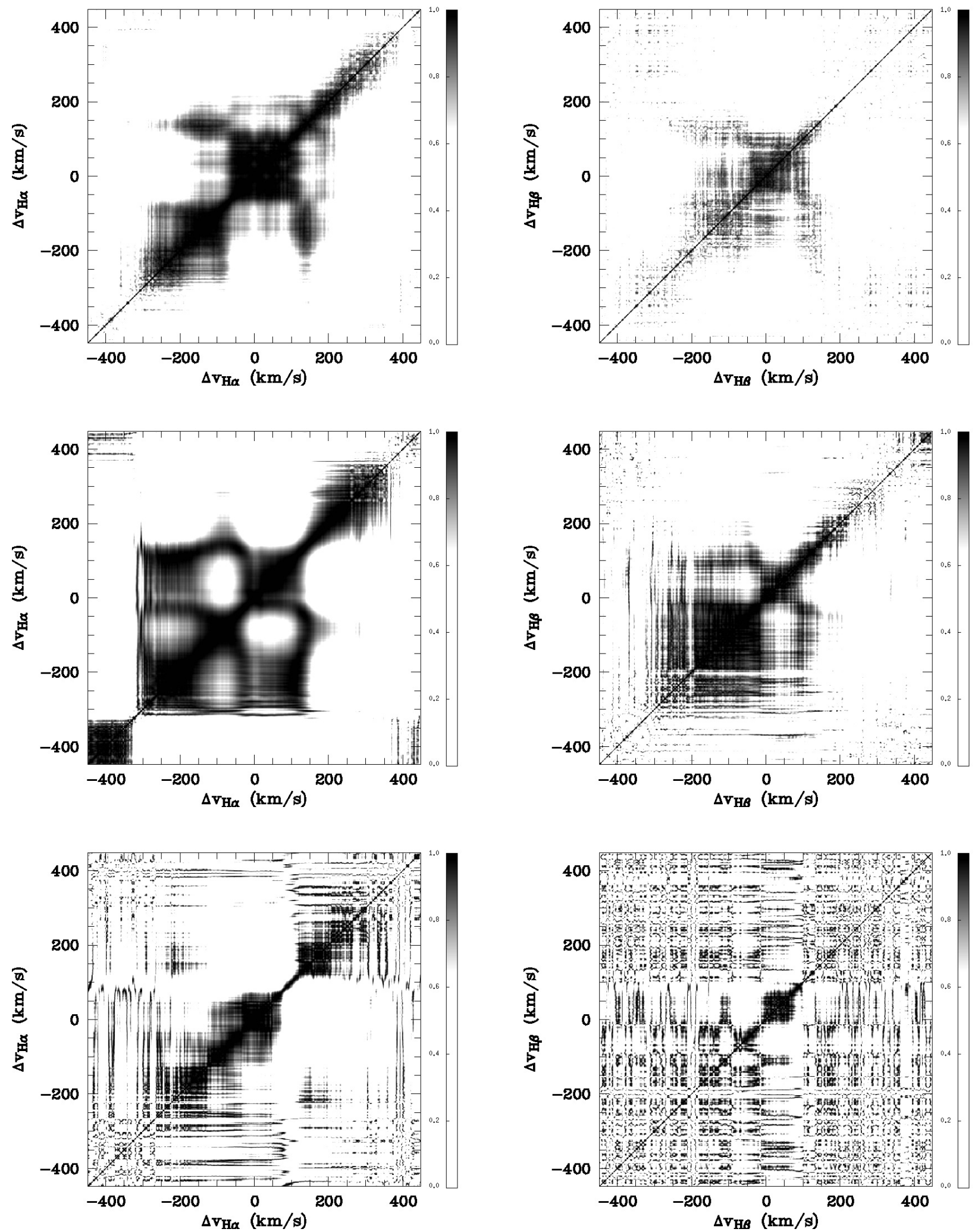

Fig. 11. Two-dimensional maps of the correlation matrices of $\mathrm{H} \alpha$ (left panels) and $\mathrm{H} \beta$ (right panels) line profiles with themselves, for the three runs of Figs. 8 and 9. Different squarish regions are distinguishable in the first two runs, indicating a coherence in the variations of $\mathrm{H} \alpha$ intensity in the velocity interval from -300 to $120 \mathrm{~km} \mathrm{~s}^{-1}$.

spectrum corresponding to the bright level as a template, an extinction of $3.3 \mathrm{mag}$ is needed to reproduce the faint spectrum, and, apart from emission lines, the residual is indeed quite low (cf. Fig. 15). 

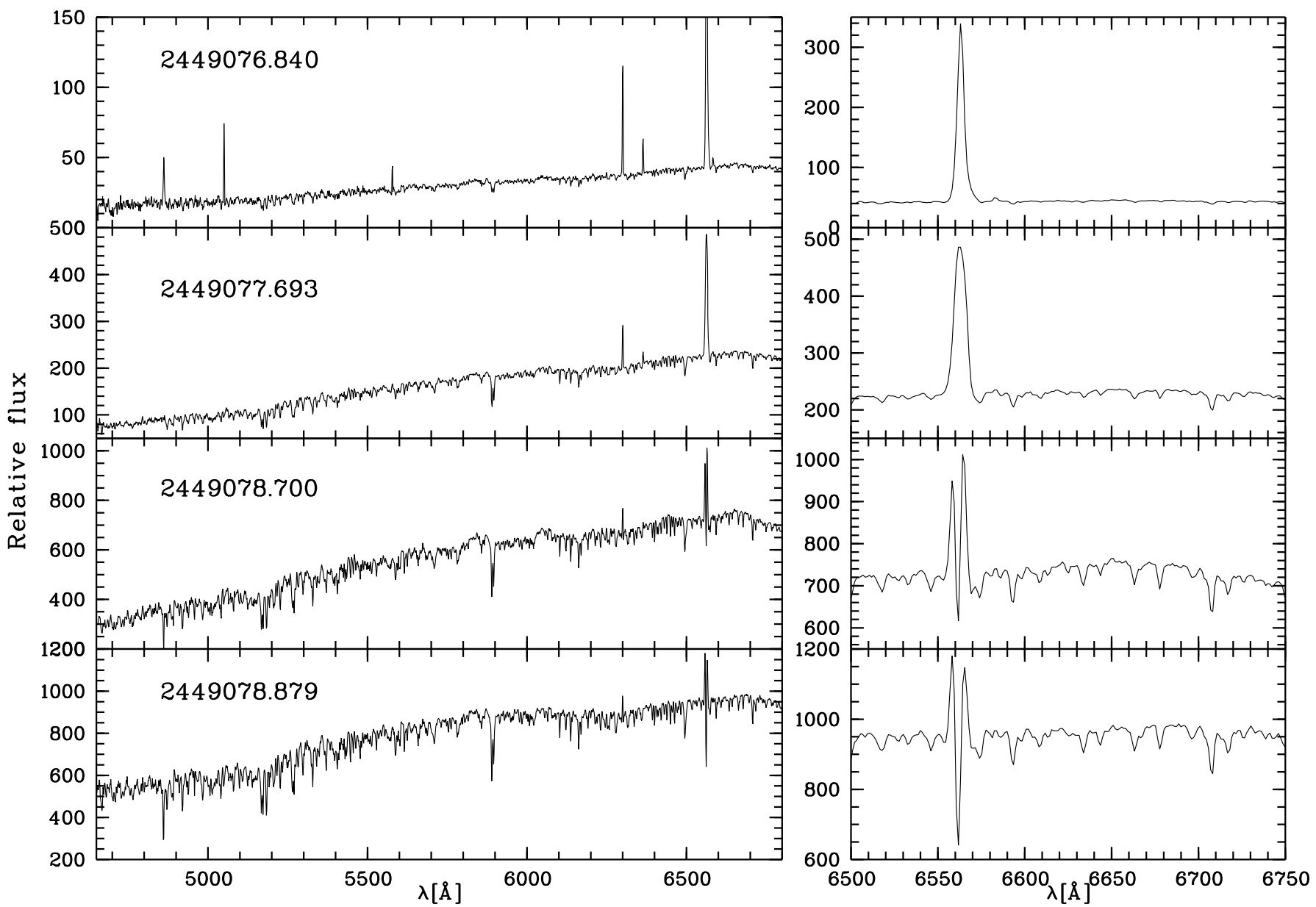

Fig. 12. Low-resolution spectra of T Cha. The panels on the left side display the whole range, those on the right show the interval containing H $\alpha$ and the Lithium line. The complete atlas of low-resolution spectra is only available electronically.

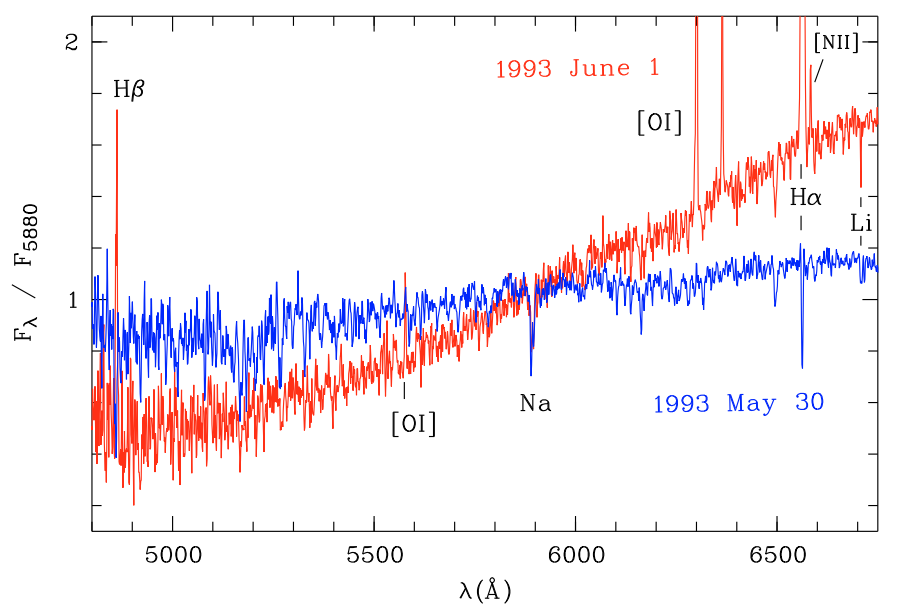

Fig. 13. Low-resolution spectra of T Cha near minimum (June 1, in red) and maximum brightness (May 30, in blue). The spectra are calibrated in relative flux and normalised to the flux at $\lambda=5880 \AA$. Emission features, only visible in the spectrum corresponding to the fainter level, are marked.

We applied the same procedure to each of the low-resolution spectra and determined the corresponding value of $A_{V}$ (see Table 4), with estimated errors of about 10\%. This takes account of the uncertainty in the fit, as well as the relative flux calibration, but not systematic effects caused by deviation of the circumstellar extinction from the normal interstellar law, as shown in Sect. 8. The two panels of Fig. 16 show that a clear trend exists between the amount of visual extinction and the intensity of the $\mathrm{H} \alpha$ and [OI] $6300 \AA$ emission lines. This indicates that the observed variations do not reflect intrinsic changes in the stellar photosphere, but arise presumably from variable circumstellar extinction, as can be inferred in Fig. 13 from the different continuum slopes of two spectra taken near maximum (on 30 May 1993) and minimum (on 1 Jun. 1993) brightness. The histogram in Fig. 17 indicates that the highest extinction events are relatively rare, while events with differential extinction below 1 mag are more frequent.

\section{The spectral energy distribution}

We constructed the observed SED of T Cha using all optical and near-IR photometry available to us (Alcalá et al. 1993; Covino et al. 1996), as well as data from public catalogues. In Fig. 18, the SED is shown for wavelengths shorter than $10 \mu \mathrm{m}$. We remark that photometric errors are smaller than the symbol size, and the considerable scatter of the optical (UBVRI bands) data points reflects the significant variability of the star. Although of smaller amplitude, variability in the near-IR ( $J H K$ bands) can also be appreciated. Therefore, relying on data that have not been acquired simultaneously may be a limitation in the SED analysis.

At the brightest level, the observed $J$ flux was lower than the expected stellar photospheric flux. However, we note that T Cha has not been monitored as extensively in the near-IR as in the 


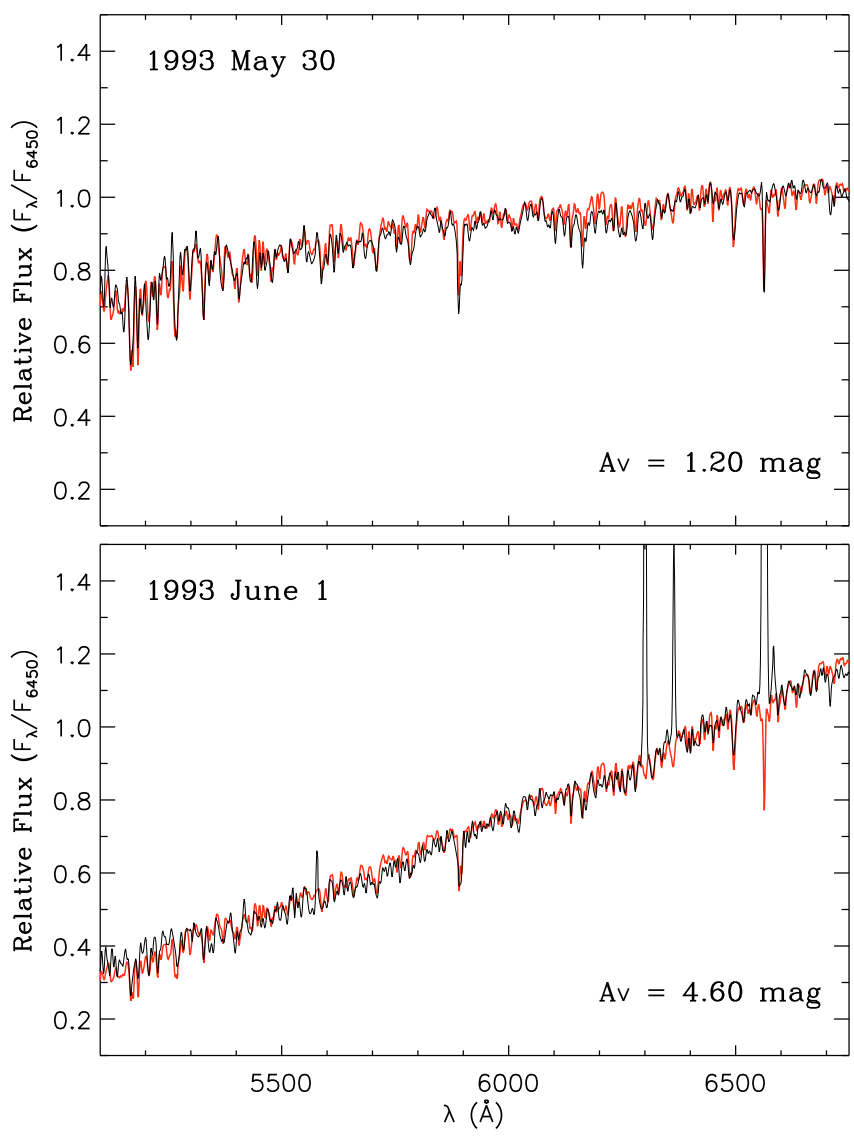

Fig. 14. Best-fit of spectral-type templates to the spectra shown in Fig. 13. The spectra of T Cha are shown as thin (black) lines, while the templates are shown as thick (red) lines. The corresponding values of total visual extinction, as derived from the two-parameter fit adopting a normal interstellar extinction law with $R_{V}=3.1$, are also indicated for both the faint and bright stage, respectively.

optical. Therefore, the available data probably did not detect the brightest $J$ flux. For that reason, the $J$ flux was excluded from our SED-fitting. Excess emission is instead observed for wavelengths longer than the $H$ band. The agreement of the Spitzer spectroscopy with the photometric data in the mid- and far-IR (cf. Fig. 19) indicates that there is no significant variability at longer wavelengths. This is expected in the case that the strong brightness and colour variations affecting the star are due to variable extinction of the stellar photosphere from inhomogeneous, circumstellar material.

By assuming variable extinction, we used relationships derived from UBVRI photometry (Covino et al. 1996) to probe the dust column affecting the brightness of T Cha. In Table 4, we report the circumstellar extinction law for $\mathrm{T} C h a$, expressed by the total to selective extinction ratios, $R_{\lambda}=A_{\lambda} / E_{B-V}$, derived from the differential brightness variations measured in the UBVRI bands (Covino et al. 1996). The resulting value of $R_{V}$ deviates from the normal value $\left(R_{V}=3.1\right)$ for the diffuse interstellar medium, which indicates different physical properties of the circumstellar grains. In particular, a higher value of $R_{V}$ means a flatter extinction curve (i.e., greyer extinction), and reveals the presence of larger grains, thus implying probable grain growth and depletion of small grains (Draine 2008).

Therefore, in the following SED analysis, we considered more suitable the flux measurements corresponding to the brightest level of the star, shown in Fig. 19.
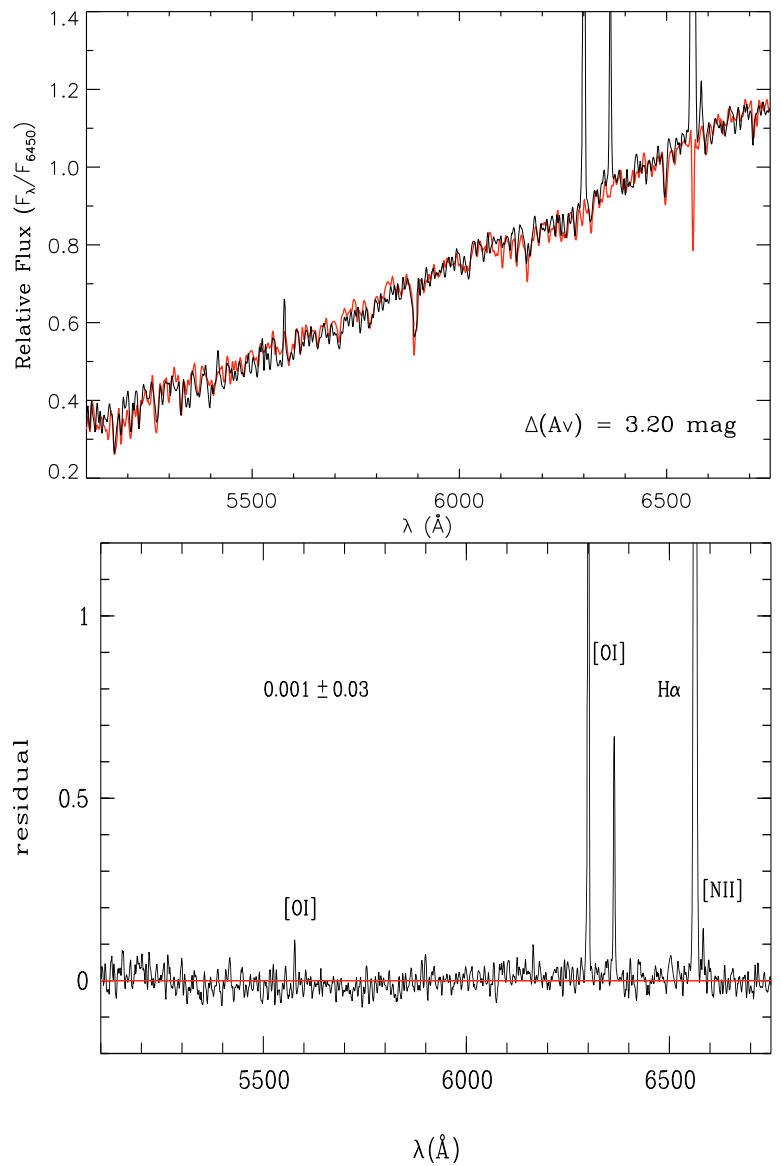

Fig. 15. The top panel shows the spectrum at the faint state as a thin (black) line, and the spectrum at the bright stage, used as a template, is shown as a thick (red) line. The lower panel shows the residual. The average residual and rms are indicated. The emission lines, not included in the deriving the average value of the residual, are marked.

Disc models such as those by Robitaille et al. (2006, 2007) do not take account of the presence of a partially evacuated inner hole or gap, so may be inadequate in reproducing the SED of transitional objects such as T Cha. Brown et al. (2007) found that the mid-IR spectrum of T Cha can be reproduced by a disc truncated at $0.08 \mathrm{AU}$ with a gap between 0.2 and $15 \mathrm{AU}$, but they did not consider the strong variability of the object nor use the available millimetre data.

We explored the possibility of modelling the SED, simultaneously from optical to millimetre wavelengths, using the CGPLUS prescription by Dullemond et al. (2001). Starting from the stellar parameters in Table 3, we constructed a grid of SEDs. The results of the SED fitting are reported in Table 3 and the best-fit models are overplotted on the observed SED in Fig. 19. The dust opacities calculated by Laor \& Draine (1993), modified to match the Beckwith et al. (1990) opacity law at wavelengths longer than $100 \mu \mathrm{m}$ were used. We note that a blackbody with $\mathrm{T}_{\text {eff }}=5400 \mathrm{~K}$ better reproduces the optical data. Previous estimates of the disc mass based on single measurements at millimetre wavelengths are in the range of $3 \times 10^{-3}-1 \times 10^{-1} M_{\odot}$ (Henning et al. 1993; Lommen et al. 2007), but our estimate reproduces well the observed data for wavelengths longer than $60 \mu \mathrm{m}$. The disc radius is more poorly constrained, but values between 100 and $150 \mathrm{AU}$ provide quite reasonable results. The latter is consistent with the $3.3 \mathrm{~mm}$ observations by Lommen et al. (2007) that did not resolve the disc around T Cha. We note that 

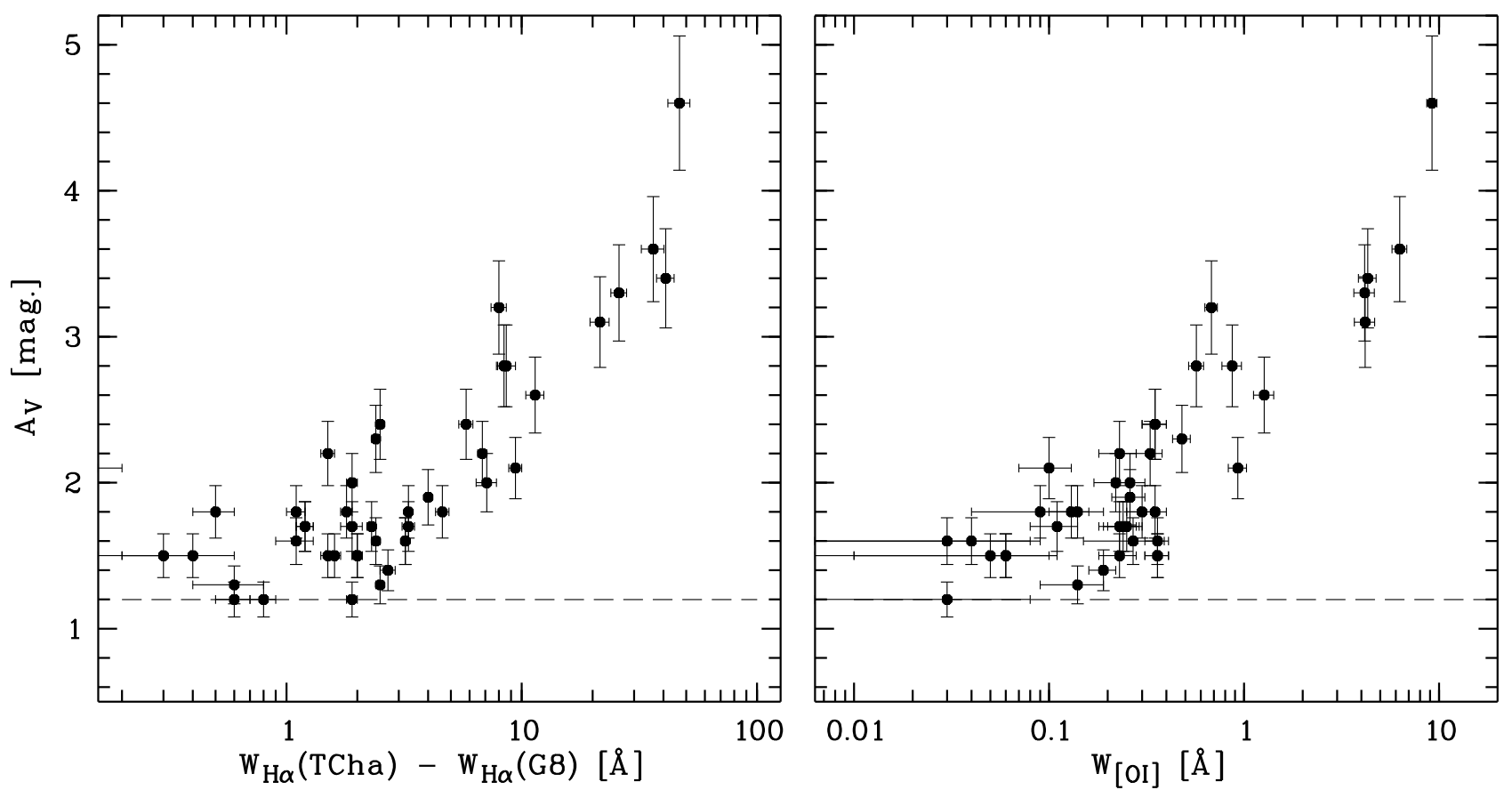

Fig. 16. Visual extinction derived from low-resolution flux-calibrated spectra versus the equivalent width of H $\alpha$ (left panel) and [OI] $6300 \AA$ (right panel) emission. For $\mathrm{H} \alpha$, the abscissa refers to the net emission after subtracting a constant value corresponding to the photospheric absorption for a G 8 star. The horizontal dashed line indicates the minimum value of $A_{V}$ obtained for T Cha at maximum brightness.

Table 4. Total to selective extinction ratios, $R_{\lambda}=A_{\lambda} / E_{B-V}$, for T Cha, derived from $U B V R I$ photometry (Covino et al. 1996).

\begin{tabular}{ccccc}
\hline \hline$R_{U}$ & $R_{B}$ & $R_{V}$ & $R_{R}$ & $R_{I}$ \\
\hline $8.4 \pm 0.4$ & $6.5 \pm 0.3$ & $5.5 \pm 0.2$ & $4.9 \pm 0.2$ & $4.5 \pm 0.2$ \\
\hline
\end{tabular}

the disc radius adopted by Brown et al. (2007) is a factor of two higher than the value derived here.

Although the model with a disc radius of 150 AU reproduces well the observed SED at optical, far-IR, and millimeter wavelengths, all the models predict too high flux between 10 and $30 \mu \mathrm{m}$ relative to the observed SED. Thus, despite our attempt to minimize the effects of colour changes by adopting the brightest flux level in each band, we were unable to fit the complete SED of T Cha with a simple reprocessing-disc model. This failure is probably caused by excess emission in the near-IR. Therefore, the most plausible disc model for reproducing the SED of T Cha is a disc with a gap, as proposed by Brown et al. (2007).

\section{Discussion and conclusions}

Before discussing our interpretation of T Cha, we briefly summarise the most significant properties of the star derived from analysis of the spectroscopic observations presented here, complemented with published photometry and other data from public archives:

a) we confirmed the spectral type G8 and a lithium abundance indicative of a very young age;

b) we detected RV variations of almost $10 \mathrm{~km} \mathrm{~s}^{-1}$ on relatively short timescale, but did not find any clear periodicity that could be related to orbital motion. Besides that, the $v \sin i$ determinations obtained from FEROS observations are consistent with the value of $37 \pm 2 \mathrm{~km} \mathrm{~s}^{-1}$, except for a few spectra in which the photospheric lines are significantly narrower, yielding a $v \sin i$ of $30 \pm 2 \mathrm{~km} \mathrm{~s}^{-1}$;

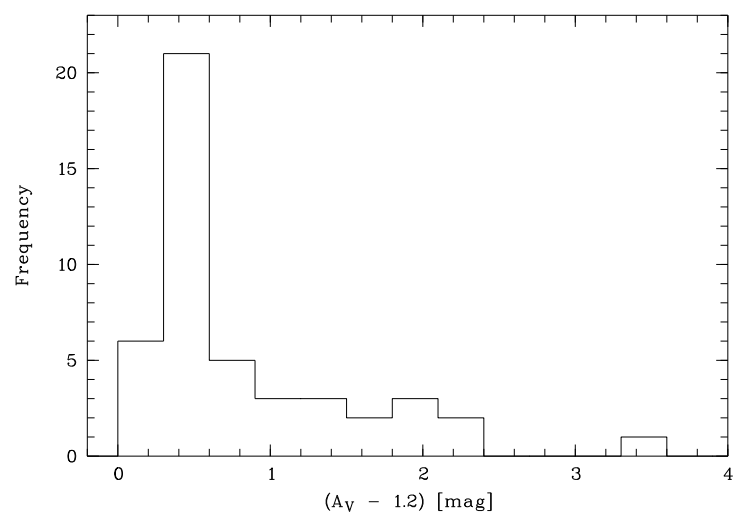

Fig. 17. Histogram of differential visual extinction. The most frequent value is around $0.5 \mathrm{mag}$, while the maximum observed is $3.3 \mathrm{mag}$.

c) strong variability in the main emission features (e.g., $\mathrm{H} \alpha, \mathrm{H} \beta$, and [OI] lines) on timescales comparable to that of photometric variability, and a close correlation between the $\mathrm{H} \alpha$ and [OI] line intensities are found;

d) variations in the photospheric continuum slope are observed in connection with intensity changes in the main emission features (i.e., the line intensities strengthen when the star reddens and fades);

e) clearly evident relationships between brightness and colours based on optical photometry (Alcalá et al. 1993; Covino et al. 1996) have allowed us to derive a circumstellar extinction law characterised by $R_{V}=5.5$ and infer the presence of large circumstellar dust grains;

f) the observed SED, showing a dip at mid-IR wavelengths, is typical of a transitional disc. SED modelling has confirmed the presence of a disc with a gap and a high inclination angle, as first indicated by Brown et al. (2007). 


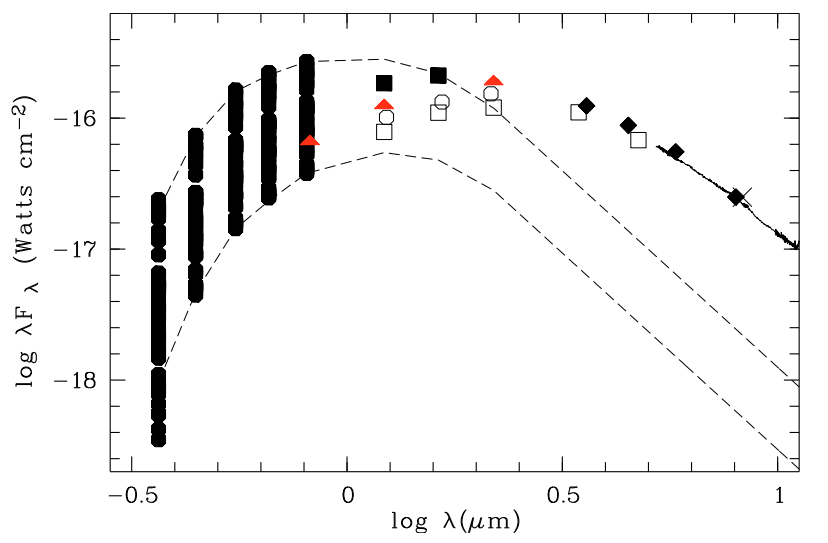

Fig. 18. Observed SED of TCha for $\lambda<1 \mu \mathrm{m}$. The $U, B, V, R$, $I$ data (filled circles) are from Alcalá et al. (1993); Covino et al. (1996). The IR fluxes come from different papers: Alcalá et al. (1993) (open squares), 2MASS fluxes (open circles), DENIS survey (filled triangles), Morel \& Magnenat Photometric Catalog (1978) (filled squares), Spitzer IRAC bands (filled diamonds), MSX6C (crosses). IRS spectra are also superposed. The upper and lower dashed lines represent the SEDs of a G8 V standard, reddened to the corresponding $(B-V)$ values that match the brightest and faintest $I$-band observed fluxes, respectively.

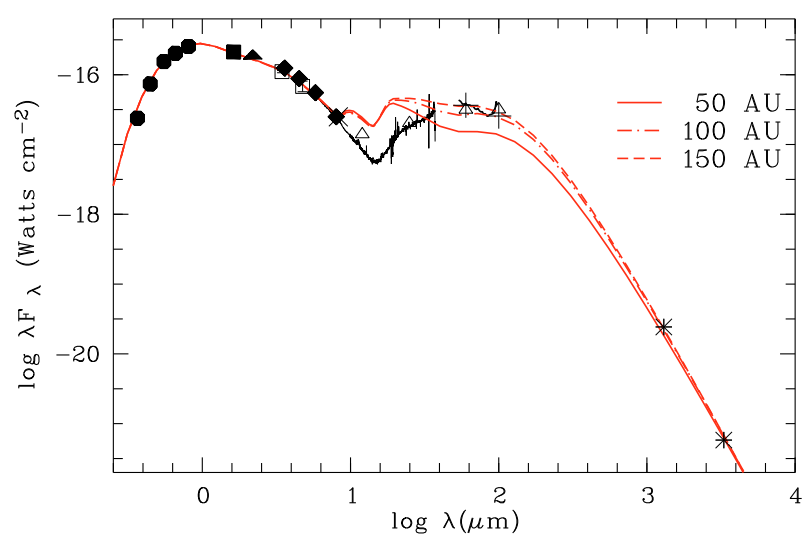

Fig. 19. Observed data-points used for SED fitting. Optical: symbols as in Fig. 18; IRAS: open triangles. Also superposed are IRS spectra (thin line). Three disc models, whose parameters are reported in Table 3, are represented by the solid, dashed and dotted lines, for different disc radii.

Several of the properties outlined above provide clues (e.g., items $c, d$, and $e$ ) that a mechanism of variable circumstellar extinction is most probably operating in TCha, as in UX Orilike stars (Natta et al. 2000; Rodgers et al. 2002). The simultaneous brightening of $\mathrm{H} \alpha$ emission and oxygen forbidden lines as reddening increases, as well as the existence of a tight correlation between brightness and colour variations, can be most plausibly explained in terms of variable extinction produced by obscuring clumpy material moving across the line of sight to the star. First of all, whereas the Hydrogen Balmer lines originate in regions closer to the star, the oxygen forbidden lines form instead farther away from the star and are hence not expected to be sensitive to variations occurring in the inner parts of the disc. Moreover, the dominant timescales and ranges of the observed variability phenomena indicate that the occulting clumps must be small compared to the size of the disc and more likely concentrated within a distance of a few tenths AU from the star. These clumps occasionally obscure the star favoured by the high inclination of the disc, while not affecting either the outer, extended, low-density wind region traced by the [OI], or the bulk of the circumstellar hydrogen emitting zone. The consequence is a strong
Table 3. In the upper table, we report the estimated stellar properties, and in the lower table, the stellar and disc parameters resulting from SED-fitting using Dullemond et al. (2001) model.

\begin{tabular}{ll}
\hline \hline Stellar Parameter & value \\
\hline$L\left(L_{\odot}\right)$ & $2.6^{\dagger}$ \\
$T_{\text {eff }}(\mathrm{K})$ & $5520^{\ddagger}$ \\
$R\left(R_{\odot}\right)$ & $1.8^{*}$ \\
$M\left(M_{\odot}\right)$ & $1.3^{* *}$ \\
Age $(\mathrm{Myr})$ & $10^{* *}$ \\
\hline Model Disc Best-Fit Parameters & \\
$L_{\star}\left(L_{\odot}\right)$ & 2.5 \\
$T_{\star}(\mathrm{K})$ & 5400 \\
$M_{\star}\left(M_{\odot}\right)$ & 1.5 \\
$M_{\text {disc }}\left(M_{\odot}\right)$ & $5 \times 10^{-3}$ \\
$R_{\text {out }}(\mathrm{AU})$ & 150 \\
$R_{\text {in }}(\mathrm{AU})$ & 0.13 \\
Temperature of rims & 1300 \\
Inclination $i$ & $75^{\circ}$ \\
\hline
\end{tabular}

$\dagger$ From SED integration and adopting $d=100 \mathrm{pc}$ and $A_{V}=1.3$; $\ddagger$ from the relations by Kenyon \& Hartmann (1995) for a G8 V star; $*$ from the Stefan-Boltzman law; ** from comparison with Palla \& Stahler (1999) PMS tracks.

contrast enhancement of all emission lines relative to the photospheric continuum when the star is highly attenuated, while the lines weaken or even disappear as the photospheric contribution turns brighter. Furthermore, these dusty clumps might also be associated with patchy gas structures orbiting close to the star $(\lessgtr 0.2 \mathrm{AU})$ that cause daily changes in the $\mathrm{H} \alpha$ line profile, similar to those we observe (see Sect. 5.3.1), due to variations in the absorbing column of material shielding the star. If the $\mathrm{H} \alpha$ variability were merely a consequence of the brightening and fading of the stellar continuum flux, the shape of the line profile should not change with time. This is generally not the case, suggesting that some other modifications in the $\mathrm{H} \alpha$ forming region must also intervene. In particular, we expect that the parts of the $\mathrm{H} \alpha$ profile originating closer to the star (e.g., the redshifted absorption components, in the case of magnetospheric accretion) also show the effects of occultation by orbiting material as well as eventual modulation induced by rotation. This is consistent with the nightly line profile changes seen in the FEROS high-resolution spectra and the continuum flux (i.e. extinction) variations sharing a common timescale. However, while we can estimate that the time required for an occulting clump to appear in front of the star likely ranges between about one-third and two-thirds of a day, it is difficult, from the present data, to quantify in a reliable way the fraction of $\mathrm{H} \alpha$ emission that may originate close to the star, as we are unable to determine information about the detailed geometry of the occultation.

But what is the source for the bulk $\mathrm{H} \alpha$ emission in T Cha? It is generally accepted that the strong $\mathrm{H} \alpha$ emission line in T Tauri stars is due to mass accretion, which leads to UV excess emission and photospheric continuum veiling (Hartigan et al. 1995). Transitional objects, however, have more evolved discs in which accretion should decrease significantly with respect to the earlier CTTS phase. In TCha, no veiling is apparently detected, not even in the stages of strong $\mathrm{H} \alpha$ emission (see Figs. 5, 15, and 20) suggesting very low or no mass accretion. However, the non-detection of veiling in T Cha may also caused by the relatively high continuum flux emitted by its G8 photosphere. Hence, we cannot exclude the possibility that erratic accretion episodes are caused by instabilities in the inner disc, in which infalling gas produces the variable redshifted 

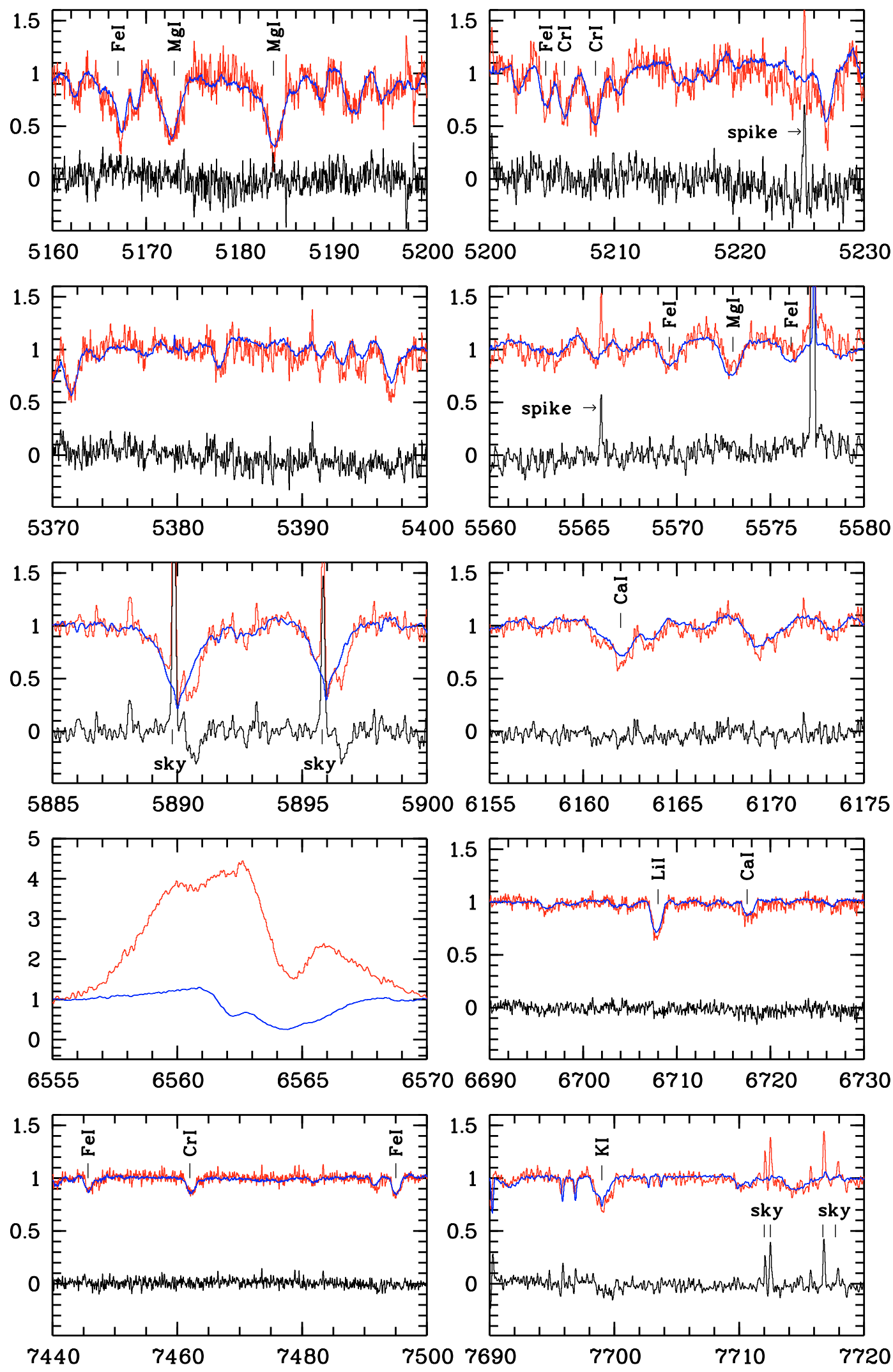

Fig. 20. Comparison between T Cha spectra observed in two different epochs: in blue the spectrum observed on 14 Feb. 2002 , in red the one observed on 26 Apr. 2004, in black the difference between the two. Various spectral lines at different wavelengths are marked.

absorption features that we observe in the $\mathrm{H} \alpha$ line profile. However, the [OI] $\lambda 6300 \AA$ emission, which corresponds to a luminosity of $\log \left(L_{[\mathrm{OI}]} / L_{\odot}\right) \approx-4.8$, implies the presence of strong winds, although the width of the line $\left(\approx 100 \mathrm{~km} \mathrm{~s}^{-1}\right.$, see
Fig. 6) is at the lower limit of a typical high-velocity component (Hartigan et al. 1995). It is probable that the bulk of the $\mathrm{H} \alpha$ emission observed in TCha is also produced in a wind, as reported for some Herbig Ae stars (Kraus et al. 2008). Moreover, the $\mathrm{H} \alpha$ 
versus [OI] correlation shown in Fig. 7 is more easily explained in terms of variable circumstellar extinction, rather than variable accretion.

By both assuming variable circumstellar extinction, and adopting the relation $A_{V}=1.086 C_{\text {ext }}(V) N_{\mathrm{H}}$ and the extinction cross-section per $\mathrm{H}$ nucleon for $R_{V}=5.5, C_{\text {ext }}(V)=6.715 \times$ $10^{-22} \mathrm{~cm}^{2} / H$, given by Draine (2003), we estimate the column density for an obscuring clump to be $N_{\mathrm{H}} \approx 1.4 \times 10^{21} A_{V} \mathrm{~cm}^{-2}$. By also assuming the maximum observed differential extinction of $3.3 \mathrm{mag}$, we obtain a hydrogen column density $N_{\mathrm{H}}=$ $4.6 \times 10^{21} \mathrm{~cm}^{-2}$. We can estimate the mass of the obscuring clumps, $M_{\mathrm{cl}}$, by assuming that they cover completely the photospheric disc of the star. Using the stellar radius reported in Table 3, the result is $M_{\mathrm{cl}} \approx 4 \times 10^{20} \mathrm{~g}$, i.e., of the order of $2 \times 10^{-13} M_{\odot}$. Similarly, assuming the most frequent differential circumstellar extinction from the histogram in Fig. 17, the result would be $M_{\mathrm{cl}} \approx 3 \times 10^{-14} M_{\odot}$. In contrast, the mass of asteroid 253 Mathilde is $5 \times 10^{-14} M_{\odot}$ (Yeomans et al. 1998).

Finally, could the RV variations in T Cha becaused by a lowmass companion? Some PMS stars with transitional discs, such as $\mathrm{CoKu} \mathrm{Tau} / 4$, have been discovered to be binaries (e.g., Ireland $\&$ Kraus 2008). The inner edge of circumbinary discs is believed to be set by dynamical truncation. In the case of CoKu Tau/4, where the binary separation is about $8 \mathrm{AU}$, the truncation radius would be of the order of 13-16 AU, depending on the eccentricity of the binary (Ireland \& Kraus 2008). As concluded from our analysis of the CCF bisector, the RV variation of T Cha is about $10 \mathrm{~km} \mathrm{~s}^{-1}$. A very low-mass object, say a $0.05 M_{\odot}$ brown dwarf on a circular orbit with a radius of about $0.1 \mathrm{AU}$ may cause this variation, producing a disc truncation radius of about $0.2 \mathrm{AU}$, consistent with the inner disc edge proposed by Brown et al. (2007). However, no obvious periodicity is detected in our RV measurements. On the other hand, the gap proposed by Brown et al. (2007) cannot be explained in terms of truncation by a companion at a separation of about 10-15 AU. At such separation, a $0.9 M_{\odot}$ star would be necessary to produce the observed RV variation. An object of that mass and separation would have been eventually detected either by us as a spectroscopic binary, or by Köhler (2001) in his speckle survey. Thus, the gap of the transitional disc in T Cha is probably not the result of dynamical truncation due to a binary companion. In any case, the presence of a lower-mass (stellar or substellar) companion cannot be definitely excluded because a quasi-periodicity of about 3 days is clearly evident throughout the entire data-set of T Cha. On the other hand, T Cha is a relatively fast rotator $\left(v \sin i \approx 37 \mathrm{~km} \mathrm{~s}^{-1}\right)$ and a magnetically active $\left(\log L_{X} \approx 30 \mathrm{erg} \mathrm{s}^{-1}\right)$ PMS star. Therefore, the observed RV variations might be alternatively induced by stellar spots, as in the case of the WTTs V410 Tau (Stelzer et al. 2003). Otherwise, we cannot exclude the possibility that the same circumstellar inhomogeneities responsible for the variable extinction may also alter the symmetry of the photospheric lines, and cause apparent RV shifts due to a sort of Rossiter effect, as in eclipsing binaries, which occurs when the stellar photosphere is partially obscured. In all cases, the presence of an inner disc characterised by a clumpy structure and the evidence of grain growth both indicate that T Cha is a likely candidate to host planet formation.

Acknowledgements. We thank the user support group of ESO/La Silla. We are grateful to Rainer Wichmann for providing us with the series of FEROS spectra of March 1999. This research has made use of SIMBAD and VIZIER databases, operated at CDS, Strasbourg, France. This publication makes use of data products from the Two Micron All Sky Survey, which is a joint project of the University of Massachusetts and the Infrared Processing and Analysis Center/California Institute of Technology, funded by the National Aeronautics and Space Administration and the National Science Foundation. This work is based in part on archivial data obtained with the Spitzer Space Telescope, which is operated by the Jet Propulsion Laboratory, California Institute of Technology under a contract with Nasa. Financial support from INAF (PRIN 2007: From active accretion to debris discs) is acknowledged. We thank the anonymous referee for constructive criticism and comments that helped to improve this paper.

\section{References}

Alcalá, J. M., Covino, E., Franchini, M., et al. 1993, A\&A, 272, 225 Alcalá, J. M., Krautter, J., Schmitt, J. H. M. M., et al. 1995, A\&AS, 114, 109 Alcalá, J. M., Spezzi, L., Frasca, A., et al. 2006, A\&A, 453, 1

Alencar, S. H. P., \& Batalha, C. 2002, ApJ, 571, 378

Alexander, R. D., \& Armitage, P. J., 2007, MNRAS, 375, 500

Appenzeller, I., \& Mundt, R. 1989, A\&ARv, 1, 291

Beckwith, S. V. W., Sargent, A. I., Chini, R. S., \& Guesten, R. 1990, AJ, 99, 924 Bertout, C. 2000, A\&A, 363,984

Bertout, C., Siess, L., \& Cabrit, S., 2007, A\&A, 473L, 21

Bessel, M. 1979, PASP, 91, 589

Bouret, J.-C., \& Catala, C. 2000, A\&A, 259, 1011

Bouvier, J., Grankin, K. N., Alencar, S. H. P., et al. 2003, A\&A, 409, 169

Brown, J. M., Blake, G. A., Dullemond, C. P., et al. 2007, ApJ, 664, 107

Calvet, N., \& Hartmann, L. 1992, ApJ, 386, 239

Calvet, N., Hartmann, L., \& Hewett, R. 1992, ApJ, 386, 229

Cardelli, J. A., Clayton, G. C., \& Mathis, J. S. 1989, AJ, 345, 245

Chiang, E. I., \& Goldreich, P. 1997, ApJ, 490, 368

Cieza, L., Padgett, D., Stapelfeldt, K. R., et al. 2007, ApJ, 667, 308

Clarke, C. J., Gendrin, A., \& Sotomayor, M. 2001, MNRAS, 328, 485

Covino, E., Terranegra, L., Franchini, M., Chavarria-K., C., \& Stalio, R. 1992, A\&AS, 94, 273

Covino, E., Alcala, J. M., Krautter, J., Terranegra, L., \& Wichmann, R. 1994, Proceedings of a Conference honoring Hans Elsässer, Disks and Outflows Around Young Stars, ed. S. V. W. Beckwith, J. Staude, A. Quetz, \& A. Natta, Lecture Notes in Physics, 465, 343

Covino, E., Alcala, J. M., Allain, S., et al. 1997, A\&A, 328, 187

Cieza, L. A. 2008, ASPC, 393, 35

D’Alessio, P., Calvet, N., Hartmann, L., Lizano, S., \& Cantó, J. 1999, ApJ, 527, 893

Dall, T. H., Santos, N. C., Arentoft, T., et al. 2006, A\&A, 454, 341

Draine, B. T. 2003, ARA\&A, 41, 241

Draine, B. T. 2008, Space Sci. Rev., in press

Dullemond, C. P., Dominik, C., \& Natta, A. 2001, ApJ, 560, 957

Dullemond, C. P., van den Ancker, M. E., Acke, B., \& van Boekel, R. 2003, ApJ, 588,360

Dullemond, C. P., \& Dominik, C. 2005, A\&A, 434, 971

Edwards, S., Hartigan, P., Ghandour, L., \& Andrulis, C. 1994, AJ, 108, 1056

Espaillat, C., Calvet, N., Luhman, K. L., Muzerolle, J., \& D’Alessio, P. 2008, ApJ, 682, 125

Esposito, M., Covino, E., Alcalá, J. M., Guenther, E. W., \& Schisano, E. 2007, MNRAS, 376, 1805

Evans, N. J. II, Dunham, M. M., Jørgensen, J. K., et al. 2008, ApJ, in press [arXiv: 0811.1059]

Franchini, M., Covino, E., Stalio, R., Terranegra, L., \& Chavarria-K., C. 1992, A\&A, 256, 525

Frink, S., Roeser, S., Alcalá, J. M., Covino, E., \& Brandner, W. 1998, A\&A, 338, 442

Furlan, E., Hartmann, L., Calvet, N., D’Alessio, P., et al. 2006, ApJS, 165, 568 Gahm, G. F., Fischerstrom, C., Lindroos, K. P., \& Liseau, R. 1989, A\&A, 211, 115

Gandolfi, D., Alcalá, L. S., et al. 2008, ApJ, 687, 1303

Geers, V. C., Augereau, J. C., Pontoppidan, K. M., et al. 2006, A\&A, 459, 545

Gras-Velazquez, A., \& Ray, T. P. 2005, A\&A, 443, 541

Greene, T. P., Wilking, B. A., André, P., et al. 1994, ApJ, 434, 614

Gray, D. F. 2005, The Observation and Analysis of Stellar Photospheres (3rd ed.: Cambridge: Cambridge Univ.)

Gregorio-Hetem, J., Lepine, J. R. D., Quast, G. R., Torres, C. A. O., \& de La Reza, R. 1992, AJ, 103, 549

Grinin, V. P. 1988, PAZh, 14,65

Guenther, E. W., Esposito, M., Mundt, R., et al. 2007, A\&A, 467, 1147

Hartmann, L., Avrett, E. H., Loeser, R., \& Calvet, N. 1990, ApJ, 349, 168

Hartigan, P., Hartmann, L., Kenyon, S., \& Hewett, R. 1989, ApJS, 70, 899

Hartigan, P., Edwards, S., \& Ghandour, L. 1995, ApJ, 452, 736

Henning, T., Pfau, W., Zinnecker, H., \& Prusti, T. 1993, A\&A, 346, 205

Herbst, W., Herbst, D. K., Grossman, E. J., \& Weinstein, D. 1994, AJ, 108, 1906

Herbst, W., \& Shevchenko, V. S. 1999, AJ, 118, 1043

Hermann, F., \& Krikov, A. V. 2007, A\&A, 829, 839

Hirth, G. A., Mundt, R., Solf, J., \& Ray, Th. P. 1994, ApJ, 427, 99 
Hoffmeister, C. 1958, Veroff. Sonnborg, 3, 342

Hollenbach, D. J., Yorke, H. W., \& Johnstone, D. 2000 in Protostars and Planets IV (Book - Tucson: University of Arizona Press), ed. V. Mannings, A. P. Boss, \& S. S. Russell, 401

Ireland., M. J., \& Kraus, A. L. 2008, ApJ, 678, 59

Isella, A., Testi, L., \& Natta, A. 2006, A\&A, 451, 951

Johns, C. M., \& Basri, G. 1995, AJ, 109, 2800

Kessler-Silacci, J., Augereau, J.-C., Dullemond, C.P., et al. 2006, ApJ, 639, 275

Kraus, S., Hofmann, K.-H., Benisty, M., et al. 2008, A\&A, 489, 1157

Kenyon, S. J., \& Hartmann, L. 1995, ApJS, 101, 117

Köhler, R. 2001, AJ, 122, 3325

Lada, C. J., Muench, A. A., Luhman, K. L., et al. 2006, AJ, 131, 1574

Laor, A., \& Draine, B. T. 1993, ApJ, 402, 441

Lommen, D., Wright, C. M., Maddison, S. T., et al. 2007, A\&A, 462, 211

Mauder, H., \& Sosna, F. M. 1975, IBVS, 1049

Merín, B., et al. 2008, in prep.

Millan-Gabet, R., et al. 2007, in Protostars \& Planets V, ed. B. Reipurth, et al. (Tucson: Univ. Arizona Press), 539

Mitskevich, A. S., Natta, A., \& Grinin, V. P. 1993, ApJ, 404, 751

Morel, M., \& Magnenat, P. 1978, Astron. Astrophys. Suppl., 34, 477

Muzzerolle, J., Calvet, N., \& Hartmann, L. 2001, ApJ, 550, 944

Najita, J. R., Stephen, E. S., \& Muzerolle, J. 2007, MNRAS, 378, 369

Natta, A., Grinin, V., \& Mannings, V. 2000, in Protostars and Planets IV (Book

- Tucson: University of Arizona Press), 559
Natta, A., Prusti, T., Neri, R, et al. 2001, A\&A, 371, 186

Natta, A., Testi, L., Muzerolle, J., et al. 2004, A\&A, 424, 603

Nordström, B., Mayor, M., Andersen, J., et al. 2004, A\&A, 418, 989

Palla, F., \& Stahler, S. W. 1999, ApJ, 525, 772

Pavlenko, Y. V., \& Magazzú, A. 1996, A\&A, 311, 961

Pickles, A. J. 1998, PASP, 110, 863

Queloz, D., Henry, G. W., Sivan, J. P., et al. 2001, A\&A, 379, 279

Reipurth, B., Pedrosa, A., \& Lago, M. T. V. T. 1996, A\&AS, 120, 229

Robitaille, T. P., Whitney, B. A., Indebetouw, R., Wood, K., \& Denzmore, P. 2006, ApJS, 167, 256

Robitaille, T. P., Whitney, B. A., Indebetouw, R., \& Wood, K. 2007, ApJS, 169, 328

Rodgers, B., Wooden, D. H., Grinin, V., Shakhovsky, D., \& Natta, A. 2002, ApJ, 564,405

Santos, N. C., Mayor, M., Naef, D., et al. 2002, A\&A, 392, 215

Scargle, J. D. 1982, ApJ, 263, 835

Shu, F., Najita, J., Ostriker, E., et al. 1994, ApJ, 429, 781

Sicilia-Aguilar, A., Hartmann, L. W., Watson, D., et al. 2007, ApJ, 659, 1637

Stelzer, B., Fernández, M., Costa, V. M., et al. 2003, A\&A, 411, 517

Terranegra, L., Morale, F., Spagna, A., Massone, G., \& Lattanzi, M. G. 1999, A\&A, 341, L79

Toner, C. G., \& Gray, D. F. 1988, ApJ, 334, 1008

Vinković, D., \& Jurkić, T. 2007, ApJ, 658, 462

White, R. J., \& Basri, G. 2003, ApJ, 582, 1109

Yeomans, D. K., Antreasian, P. G., Barriot, J.-P., et al. 1998, Science, 289, 2085 
E. Schisano et al.: Variability of T Cha, Online Material $p 1$

Table 1. Journal of FEROS observations and radial velocity measurements.

\begin{tabular}{|c|c|c|c|c|c|c|c|}
\hline Date & $\begin{array}{c}\text { HJD } \\
-2400000 .\end{array}$ & $\begin{array}{c}\mathrm{RV}_{g} \\
\left(\mathrm{~km} \mathrm{~s}^{-1}\right)\end{array}$ & $\begin{array}{c}\sigma_{\mathrm{RV}} \\
\left(\mathrm{km} \mathrm{s}^{-1}\right)\end{array}$ & Date & $\begin{array}{c}\text { HJD } \\
-240000 .\end{array}$ & $\begin{array}{c}\mathrm{RV}_{g} \\
\left(\mathrm{~km} \mathrm{~s}^{-1}\right)\end{array}$ & $\begin{array}{c}\sigma_{\mathrm{RV}} \\
\left(\mathrm{km} \mathrm{s}^{-1}\right)\end{array}$ \\
\hline 27 March 1999 & 51265.537 & 14.27 & 0.27 & 22 May 2000 & 51687.648 & 22.97 & 1.77 \\
\hline 27 March 1999 & 51265.576 & 16.89 & 0.68 & 23 May 2000 & 51688.640 & 9.82 & 0.31 \\
\hline 28 March 1999 & 51265.662 & 15.09 & 1.63 & 04 January 2001 & 51913.860 & 13.86 & 2.13 \\
\hline 28 March 1999 & 51266.573 & 18.21 & 0.73 & 09 January 2001 & 51918.775 & 15.65 & 2.15 \\
\hline 29 March 1999 & 51266.636 & 21.39 & 0.68 & 14 January 2001 & 51923.824 & 14.89 & 0.56 \\
\hline 29 March 1999 & 51266.672 & 21.30 & 0.90 & 19 April 2001 & 52019.578 & 16.00 & 1.81 \\
\hline 30 March 1999 & 51267.692 & 14.09 & 0.25 & 26 April 2001 & 52026.586 & 14.48 & 1.12 \\
\hline 30 March 1999 & 51267.801 & 17.88 & 0.47 & 26 April 2001 & 52031.573 & 14.43 & 0.28 \\
\hline 30 March 1999 & 51267.874 & 18.01 & 1.38 & 14 February 2002 & 52319.745 & 12.63 & 0.36 \\
\hline 31 March 1999 & 51268.624 & 11.32 & 0.87 & 17 February 2002 & 52322.742 & 27.48 & 2.56 \\
\hline 31 March 1999 & 51268.727 & 16.21 & 0.86 & 08 April 2002 & 52372.606 & 14.70 & - \\
\hline 31 March 1999 & 51268.850 & 6.43 & 2.01 & 21 April 2002 & 52385.529 & 16.44 & 0.87 \\
\hline 31 March 1999 & 51268.900 & 12.20 & 1.44 & 01 May 2002 & 52395.617 & 4.33 & 1.22 \\
\hline 01 April 1999 & 51269.657 & 12.05 & 1.15 & 02 May 2002 & 52396.614 & 9.93 & - \\
\hline 01 April 1999 & 51269.771 & 13.09 & 1.00 & 12 March 2003 & 52710.601 & 21.73 & 0.30 \\
\hline 01 April 1999 & 51269.857 & 15.80 & 1.56 & 12 March 2003 & 52717.620 & 14.13 & 1.51 \\
\hline 16 Маy 1999 & 51315.622 & 18.17 & 0.76 & 25 March 2003 & 52723.588 & 15.96 & 2.33 \\
\hline 17 May 1999 & 51316.612 & 6.43 & 0.82 & 02 April 2004 & 53097.541 & 15.57 & 0.87 \\
\hline 18 May 1999 & 51317.627 & 16.67 & 1.77 & 09 April 2004 & 53104.652 & 11.28 & 1.18 \\
\hline 19 May 1999 & 51318.591 & 13.50 & 1.72 & 26 April 2004 & 53122.685 & 15.93 & 0.48 \\
\hline 21 May 1999 & 51320.591 & 17.78 & 0.52 & 10 May 2004 & 53137.672 & 22.23 & 0.66 \\
\hline 22 May 1999 & 51321.580 & 12.72 & 2.92 & 10 May 2005 & 53501.652 & 29.06 & 0.87 \\
\hline 09 May 2000 & 51674.670 & 18.52 & 0.46 & 02 June 2005 & 53524.597 & 6.63 & 1.35 \\
\hline 19 May 2000 & 51684.617 & 9.90 & 0.52 & 30 June 2005 & 53552.546 & 14.31 & 1.09 \\
\hline 21 May 2000 & 51686.632 & 21.19 & 2.59 & 19 August 2005 & 53602.488 & 29.51 & 1.95 \\
\hline
\end{tabular}


E. Schisano et al.: Variability of T Cha, Online Material p 2

Table 2. Radial velocity determinations, both from gaussian fit and bisector method, and equivalent widths of H $\alpha$ and [OI] $6300 \AA$ measured on FEROS spectra.

\begin{tabular}{|c|c|c|c|c|c|c|c|c|c|c|}
\hline $\begin{array}{l}\text { HJD } \\
-2450000\end{array}$ & $\begin{array}{r}\mathrm{RV}_{g} \\
\left(\mathrm{~km} \mathrm{~s}^{-1}\right)\end{array}$ & $\begin{array}{c}\mathrm{RV}_{g} \\
\left(\mathrm{~km} \mathrm{~s}^{-1}\right)\end{array}$ & $\begin{array}{r}\mathrm{RV}_{b} \\
\left(\mathrm{~km} \mathrm{~s}^{-1}\right)\end{array}$ & $\begin{array}{c}\mathrm{RV}_{b} \\
\left(\mathrm{~km} \mathrm{~s}^{-1}\right)\end{array}$ & $\begin{array}{l}W_{\mathrm{H} \alpha} \\
(\AA)\end{array}$ & $\begin{array}{l}\sigma_{\mathrm{H} \alpha} \\
(\AA)\end{array}$ & $\begin{array}{r}\mathrm{W}_{\mathrm{H} \beta} \\
(\AA)\end{array}$ & $\begin{array}{l}\sigma_{\mathrm{H} \beta} \\
(\AA)\end{array}$ & $\begin{array}{r}W_{6300} \\
(\AA)\end{array}$ & $\begin{array}{c}\sigma_{6300} \\
(\AA)\end{array}$ \\
\hline 51265.537 & 14.27 & 0.27 & 14.64 & 1.07 & -6.26 & 1.06 & -1.37 & 0.78 & -0.81 & 0.05 \\
\hline 51265.576 & 16.89 & 0.68 & 16.54 & 1.14 & -6.56 & 0.50 & -0.34 & 0.29 & -1.00 & 0.14 \\
\hline 51265.662 & 15.09 & 1.63 & 17.21 & 0.61 & -7.50 & 0.30 & -0.56 & 0.24 & -1.01 & 0.11 \\
\hline 51266.573 & 18.21 & 0.73 & 17.81 & 0.54 & -10.64 & 0.42 & -2.00 & 0.29 & -1.34 & 0.01 \\
\hline 51266.636 & 21.39 & 0.68 & 20.57 & 1.81 & -12.46 & 0.37 & -2.02 & 0.38 & -1.48 & 0.15 \\
\hline 51266.672 & 21.30 & 0.90 & 20.70 & 1.98 & -12.36 & 0.80 & -2.89 & 0.32 & -1.56 & 0.11 \\
\hline 51267.692 & 14.09 & 0.25 & 14.35 & 2.16 & -2.89 & 0.39 & -0.01 & 0.21 & -0.58 & 0.12 \\
\hline 51267.801 & 17.88 & 0.47 & 17.58 & 0.48 & -2.72 & 0.26 & 0.03 & 0.23 & -0.43 & 0.06 \\
\hline 51267.874 & 18.01 & 1.38 & 17.81 & 0.82 & -2.55 & 0.29 & 0.03 & 0.22 & -0.34 & 0.03 \\
\hline 51268.624 & 11.32 & 0.87 & 15.40 & 1.88 & -2.83 & 0.36 & 0.32 & 0.23 & -0.40 & 0.03 \\
\hline 51268.727 & 16.21 & 0.86 & 17.10 & 1.16 & -5.32 & 0.35 & -0.41 & 0.20 & -0.39 & 0.05 \\
\hline 51268.850 & 6.43 & 2.01 & 18.39 & 1.29 & -5.05 & 0.41 & 0.07 & 0.25 & -0.35 & 0.04 \\
\hline 51268.900 & 12.20 & 1.44 & 18.75 & 2.07 & -3.96 & 0.35 & 0.10 & 0.36 & -0.39 & 0.03 \\
\hline 51269.657 & 12.05 & 1.15 & 12.76 & 1.46 & -6.18 & 0.47 & -0.31 & 0.28 & -0.78 & 0.05 \\
\hline 51269.771 & 13.09 & 1.00 & 13.42 & 0.91 & -5.19 & 0.63 & -0.76 & 0.40 & -0.77 & 0.11 \\
\hline 51269.857 & 15.80 & 1.56 & 16.56 & 0.29 & -4.23 & 0.55 & -0.95 & 0.44 & -0.80 & 0.17 \\
\hline 51315.622 & 18.17 & 0.76 & 17.65 & 0.83 & -22.68 & 0.39 & -2.88 & 0.51 & -1.88 & 0.25 \\
\hline 51316.612 & 6.43 & 0.82 & 10.54 & 3.80 & -23.93 & 0.95 & -5.89 & 0.70 & -2.06 & 0.22 \\
\hline 51317.627 & 16.67 & 1.77 & 20.90 & 1.78 & -20.20 & 1.03 & -4.30 & 0.39 & -0.88 & 0.06 \\
\hline $51318.591^{\dagger}$ & 13.50 & 1.72 & 13.42 & 0.49 & -20.65 & 0.28 & -3.03 & 0.32 & -1.22 & 0.07 \\
\hline 51320.591 & 17.78 & 0.52 & 12.61 & 2.59 & -1.42 & 0.30 & 1.08 & 0.17 & -0.28 & 0.03 \\
\hline 51321.580 & 12.72 & 2.92 & 9.64 & 2.72 & -7.56 & 0.55 & -0.07 & 0.29 & -0.64 & 0.04 \\
\hline 51674.670 & 18.52 & 0.46 & 17.39 & 1.38 & -0.75 & 0.38 & 0.87 & 0.67 & -0.35 & 0.03 \\
\hline 51684.617 & 9.90 & 0.52 & 10.45 & 1.69 & -2.39 & 0.34 & 0.43 & 0.25 & -0.69 & 0.03 \\
\hline 51686.632 & 21.19 & 2.59 & 19.51 & 2.50 & -1.13 & 0.22 & 0.59 & 0.39 & -0.52 & 0.04 \\
\hline 51687.648 & 22.97 & 1.77 & 19.71 & 2.94 & -0.88 & 0.21 & 0.86 & 0.29 & -0.39 & 0.03 \\
\hline 51688.640 & 9.82 & 0.31 & 11.37 & 3.17 & -2.34 & 0.35 & -0.06 & 0.43 & -0.48 & 0.07 \\
\hline 51913.860 & 13.86 & 2.13 & 13.69 & 1.88 & -1.46 & 0.41 & 0.55 & 0.19 & -0.23 & 0.04 \\
\hline 51918.775 & 15.65 & 2.15 & 15.90 & 0.43 & -1.86 & 0.29 & 0.15 & 0.23 & -0.22 & 0.04 \\
\hline 51923.824 & 14.89 & 0.56 & 14.91 & 0.54 & -1.12 & 0.40 & -0.20 & 0.17 & -0.14 & 0.01 \\
\hline 52019.578 & 16.00 & 1.81 & 16.07 & 0.97 & -23.82 & 0.45 & -4.06 & 1.09 & -1.83 & 0.02 \\
\hline 52026.586 & 14.48 & 1.12 & 15.03 & 1.05 & -3.80 & 0.40 & -0.26 & 0.26 & -0.55 & 0.05 \\
\hline 52031.573 & 14.43 & 0.28 & 14.64 & 0.95 & -9.18 & 0.68 & -1.45 & 0.30 & -0.64 & 0.04 \\
\hline 52319.745 & 12.63 & 0.36 & 12.66 & 1.40 & 0.26 & 0.31 & 1.22 & 0.12 & -0.26 & 0.04 \\
\hline $52322.742^{\dagger}$ & 27.48 & 2.56 & 19.18 & 3.41 & -8.56 & 0.40 & -1.23 & 0.75 & -1.02 & 0.06 \\
\hline 52372.606 & 14.70 & - & 9.92 & 2.40 & -29.09 & 0.95 & -5.60 & 0.55 & -2.33 & 0.18 \\
\hline 52385.529 & 16.44 & 0.87 & 16.54 & 0.62 & -3.82 & 0.32 & -0.60 & 0.31 & -0.49 & 0.02 \\
\hline 52395.617 & 4.33 & 1.22 & 9.46 & 2.23 & -20.92 & 1.11 & -5.22 & 0.71 & -1.97 & 0.11 \\
\hline 52396.614 & 9.93 & - & 16.20 & 3.49 & -11.51 & 1.29 & -0.50 & 0.50 & -1.78 & 0.23 \\
\hline 52710.601 & 21.73 & 0.30 & 20.00 & 1.93 & -0.93 & 0.22 & 0.57 & 0.16 & -0.31 & 0.03 \\
\hline 52717.620 & 14.13 & 1.51 & 15.79 & 1.78 & -13.51 & 0.45 & -2.06 & 0.50 & -1.16 & 0.07 \\
\hline 52723.588 & 15.96 & 2.33 & 15.80 & 0.51 & -2.09 & 0.31 & 0.62 & 0.47 & -0.35 & 0.01 \\
\hline 53097.541 & 15.57 & 0.87 & 19.17 & 1.55 & -6.14 & 0.34 & -0.18 & 0.25 & -0.72 & 0.02 \\
\hline 53104.652 & 11.28 & 1.18 & 11.38 & 2.22 & -0.93 & 0.21 & 0.77 & 0.15 & -0.20 & 0.10 \\
\hline 53122.685 & 15.93 & 0.48 & 11.70 & 3.40 & -22.39 & 0.50 & -3.59 & 1.23 & -2.00 & 0.27 \\
\hline 53137.672 & 22.23 & 0.66 & 15.42 & 0.86 & -4.21 & 0.21 & 0.40 & 0.29 & -0.23 & 0.11 \\
\hline 53501.652 & 29.06 & 0.87 & 16.43 & 1.11 & -8.22 & 0.34 & -1.23 & 0.67 & -0.59 & 0.06 \\
\hline 53524.597 & 6.63 & 1.35 & 7.95 & 2.57 & -5.55 & 0.27 & -0.18 & 0.41 & -0.51 & 0.02 \\
\hline 53552.546 & 14.31 & 1.09 & 14.36 & 0.37 & -1.06 & 0.25 & -0.04 & 0.23 & -0.22 & 0.04 \\
\hline 53602.488 & 29.51 & 1.95 & 20.32 & 4.58 & -8.66 & 0.34 & -0.77 & 0.60 & -0.68 & 0.05 \\
\hline
\end{tabular}

$\dagger$ He I $5876 \AA$ A detected. 
Table 4. Equivalent widths of $\mathrm{H} \alpha$ and [OI] $6300 \AA$ A measured on the lowresolution spectra (see the atlas in electronic form) and corresponding total extinction, $A_{V}$, obtained as described in Sect. 8.

\begin{tabular}{|c|c|c|c|c|c|c|}
\hline Date & $\begin{array}{c}\text { JD } \\
-2440000 \\
\end{array}$ & $\begin{array}{r}W_{\mathrm{H} \alpha} \\
(\AA) \\
\end{array}$ & $\begin{array}{r}\sigma_{\mathrm{H} \alpha} \\
(\AA) \\
\end{array}$ & $\begin{array}{r}W_{[O I]} \\
(\AA) \\
\end{array}$ & $\begin{array}{r}\sigma_{[O I]} \\
(\AA) \\
\end{array}$ & $\begin{array}{c}A_{V} \\
\text { (mag) }\end{array}$ \\
\hline 07 May 92 & 8749.668 & -4.58 & 0.30 & -0.33 & 0.05 & 2.2 \\
\hline 08 May 92 & 8750.606 & -3.63 & 0.40 & -0.35 & 0.05 & 2.4 \\
\hline 10 May 92 & 8752.628 & 0.40 & 0.10 & -0.13 & 0.03 & 1.8 \\
\hline 11 May 92 & 8753.634 & -4.87 & 0.70 & -0.22 & 0.05 & 2.0 \\
\hline 12 May 92 & 8754.600 & -6.42 & 0.80 & -0.87 & 0.10 & 2.8 \\
\hline $27 \mathrm{M}$ & 9073 & -1.09 & 0.20 & -0.23 & 0.05 & 1.7 \\
\hline 27 Mar. 93 & 9073.847 & -0.51 & 0.20 & -0.19 & 0.03 & 1.4 \\
\hline 28 Mar. 93 & 9074.689 & 1.80 & 0.20 & - & - & 1.5 \\
\hline 28 Mar. 93 & 9074.843 & 2.08 & 0.20 & -0.06 & 0.05 & 1.5 \\
\hline 29 Mar. 93 & 9075.680 & 0.28 & 0.20 & -0.25 & 0.05 & 1.7 \\
\hline 29 Mar. 93 & 9075.873 & -0.14 & 0.10 & -0.11 & 0.03 & 1.7 \\
\hline 30 Mar. 93 & 9076.758 & -23.70 & 2.00 & -4.16 & 0.50 & 3.3 \\
\hline 30 Mar. 93 & 9076.840 & -34.00 & 4.00 & -6.29 & 0.55 & 3.6 \\
\hline 31 Mar. 93 & 9077.693 & -9.20 & 1.00 & -1.27 & 0.15 & 2.6 \\
\hline 01 Apr. 93 & 9078.700 & -1.80 & 0.10 & -0.26 & 0.05 & 1.9 \\
\hline 01 Apr. 93 & 9078.879 & -0.32 & 0.10 & -0.14 & 0.05 & 1.3 \\
\hline 02 Apr. 93 & 9079.687 & 1.90 & 0.10 & -0.06 & 0.05 & 1.5 \\
\hline 02 Apr. & 9079.879 & 1.64 & 0.10 & - & - & 1.2 \\
\hline $30 \mathrm{M}$ & 9137.531 & 1.40 & 0.10 & - & - & 1.2 \\
\hline 31 May & 9138.515 & 0.26 & 0.10 & -0.26 & 0.05 & 2.0 \\
\hline 31 May 93 & 9138.738 & -0.25 & 0.10 & -0.35 & 0.05 & 2.4 \\
\hline $01 \mathrm{Jun}$ & 9139.545 & -44.60 & 5.00 & -9.20 & 0.55 & 4.6 \\
\hline $03 \mathrm{Ju}$ & 9142.495 & 0.16 & 0.10 & -0.36 & 0.05 & 1.5 \\
\hline 04 Jun. 93 & 9143.494 & -0.23 & 0.10 & -0.48 & 0.05 & 2.3 \\
\hline 05 Jun. 93 & 9143.736 & 0.66 & 0.10 & -0.23 & 0.05 & 2.2 \\
\hline 05 Jun. 93 & 9144.496 & -6.20 & 0.50 & -0.57 & 0.05 & 2.8 \\
\hline 06 Jun. 93 & 9144.718 & -5.81 & 0.60 & -0.68 & 0.05 & 3.2 \\
\hline 28 Jun. 93 & 9167.463 & -2.40 & 0.30 & -0.35 & 0.05 & 1.8 \\
\hline 29 Jun. 93 & 9168.478 & -19.3 & 2.00 & -4.18 & 0.50 & 3.1 \\
\hline $02 \mathrm{Jul}$ & 9170.519 & -7.21 & 0.60 & -0.93 & 0.10 & 2.1 \\
\hline $03 \mathrm{Ju}$ & 9172. & -57.0 & 5.50 & -9.00 & 0.60 & $\ddagger$ \\
\hline $24 \mathrm{M}$ & 9435.7 & 1.73 & 0.10 & -0.09 & 0.05 & 1.8 \\
\hline $24 \mathrm{M}$ & 9435.8 & 2.20 & 0.20 & -0.10 & 0.03 & 2.1 \\
\hline $25 \mathrm{~N}$ & 9436. & 0.55 & 0.10 & -0.23 & 0.05 & 1.5 \\
\hline & & -1.03 & 0.10 & -0.36 & 0.05 & 1.6 \\
\hline 26 & 943 & 0.96 & 0.10 & -0.24 & 0.05 & 1.7 \\
\hline $26 \mathrm{M}$ & 943 & 0.21 & 0.10 & -0.36 & 0.05 & 1.5 \\
\hline 7 Mar. 94 & 9438.519 & -1.10 & 0.10 & -0.30 & 0.05 & 1.8 \\
\hline 27 Mar. 94 & 9438.662 & -0.95 & 0.10 & -0.27 & 0.12 & 1.6 \\
\hline 28 Mar. 94 & 9439.520 & 1.61 & 0.20 & - & - & 1.3 \\
\hline 28 Mar. 94 & 9439.661 & 0.95 & 0.10 & - & - & 1.7 \\
\hline 29 Mar. 94 & 9440.518 & 0.25 & 0.10 & -0.03 & 0.05 & 1.2 \\
\hline 29 Mar. 94 & 9440.661 & 1.05 & 0.20 & -0.03 & 0.05 & 1.6 \\
\hline 09 Apr. 95 & 9816.807 & -0.19 & 0.10 & -0.04 & 0.05 & 1.6 \\
\hline 10 Apr. 95 & 9817.789 & -38.70 & 3.50 & -4.31 & 0.45 & 3.4 \\
\hline 11 Apr. 95 & 9818.805 & 0.70 & 0.10 & -0.05 & 0.05 & 1.5 \\
\hline 12 Apr. 95 & 9819.788 & 1.05 & 0.10 & -0.14 & 0.05 & 1.8 \\
\hline
\end{tabular}

$\ddagger \mathrm{A}_{V}$ not determined due to very faint continuum. 
E. Schisano et al.: Variability of T Cha, Online Material p 4
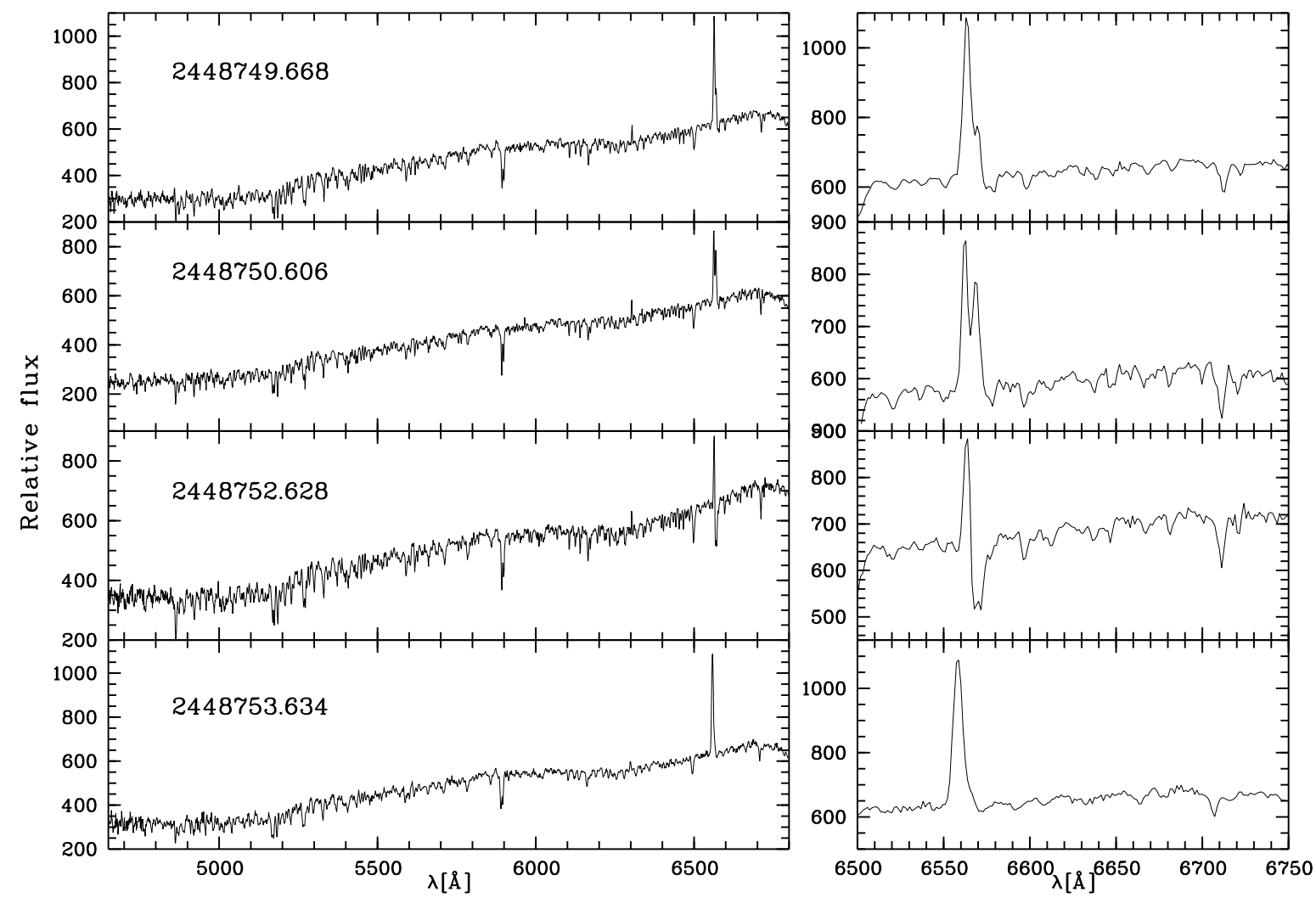

Fig. 21. Atlas of low-resolution spectra of T Cha. The panels on the left side display the whole range, those on the right show the interval containing $\mathrm{H} \alpha$ and the Lithium line. 
E. Schisano et al.: Variability of T Cha, Online Material p 5
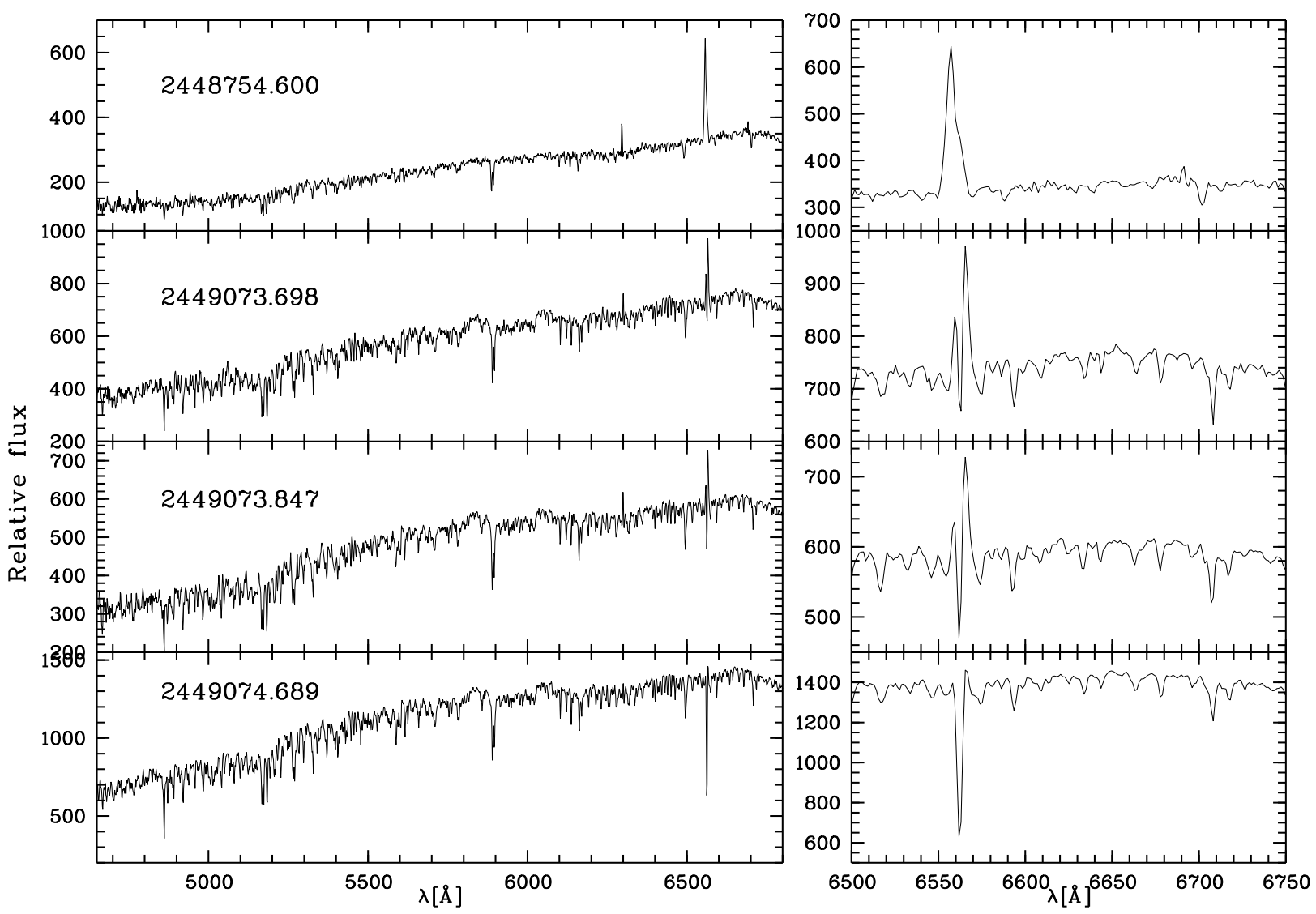

Fig. 22. Atlas of low-resolution spectra of T Cha, continue.
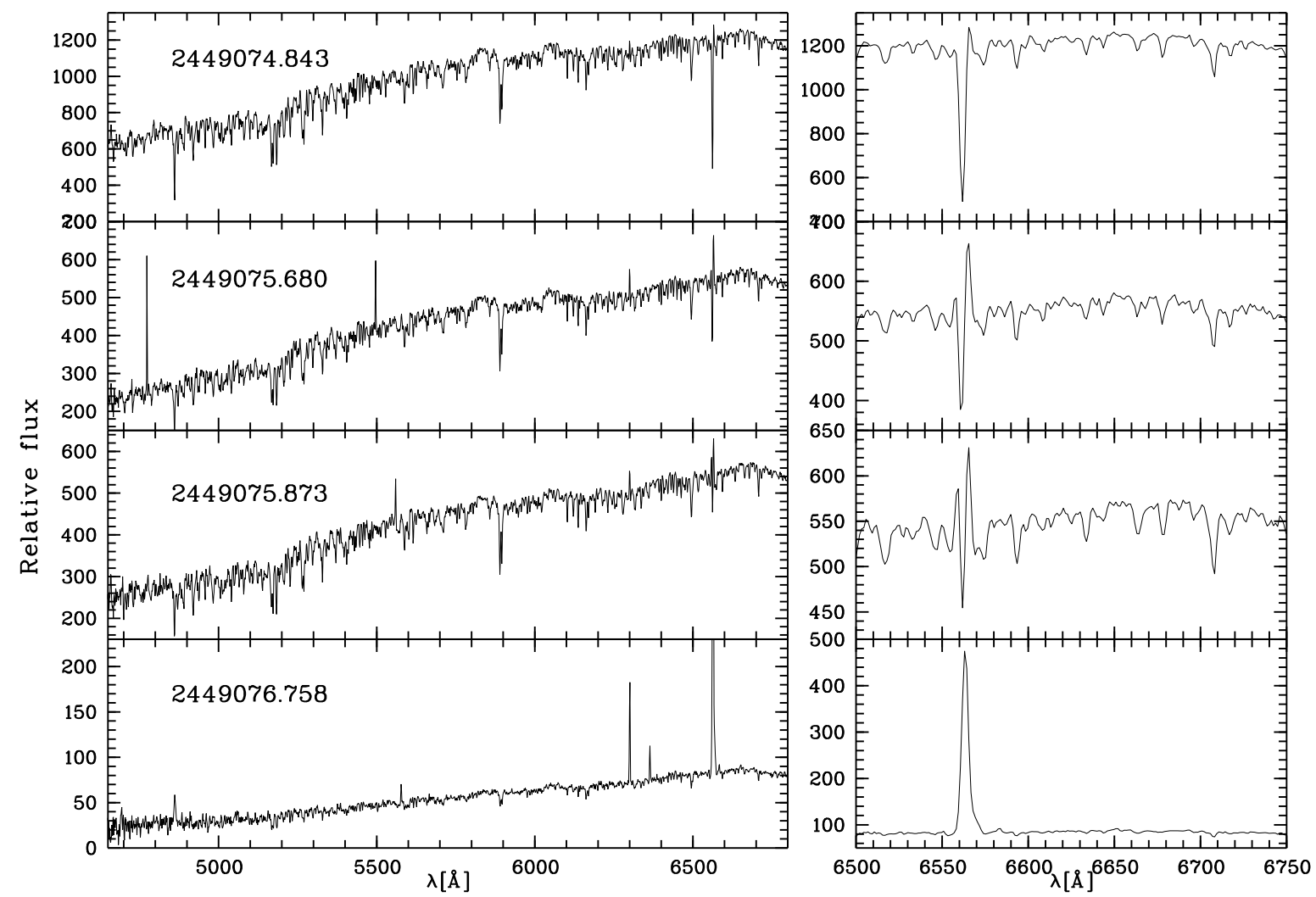

Fig. 23. Atlas of low-resolution spectra of T Cha, continue. 
E. Schisano et al.: Variability of T Cha, Online Material p 6
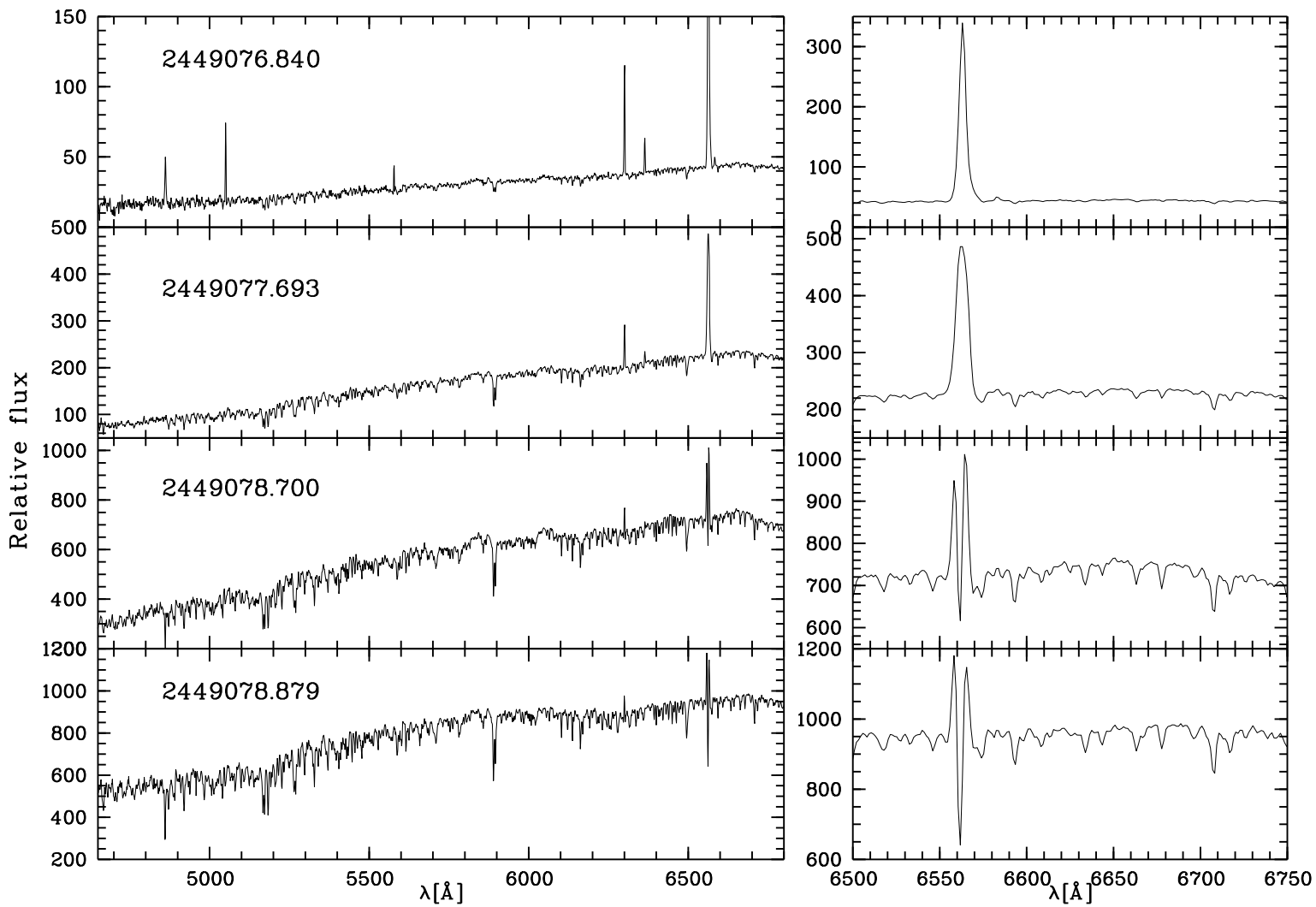

Fig. 24. Atlas of low-resolution spectra of T Cha, continue.
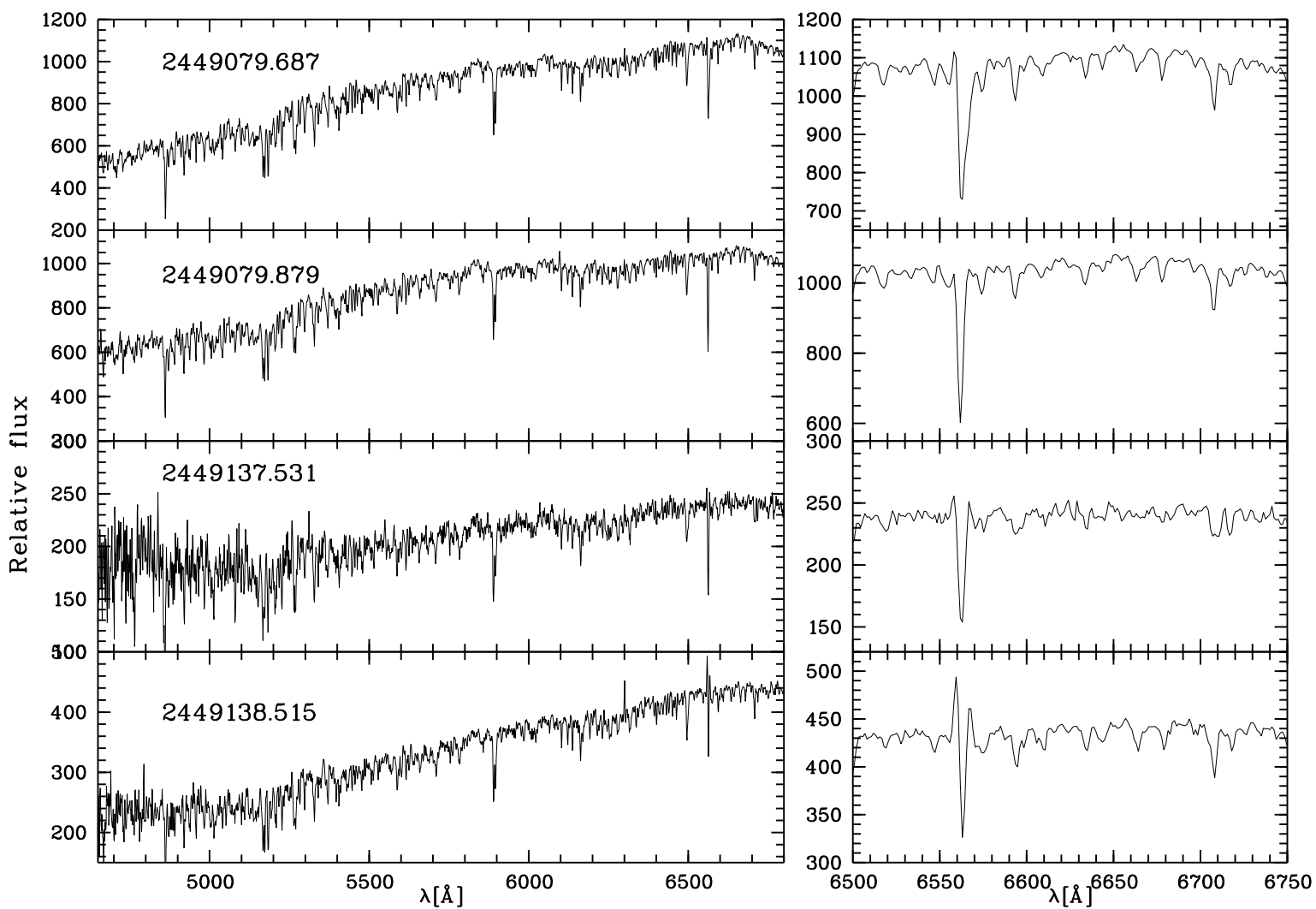

Fig. 25. Atlas of low-resolution spectra of T Cha, continue. 
E. Schisano et al.: Variability of T Cha, Online Material $p 7$
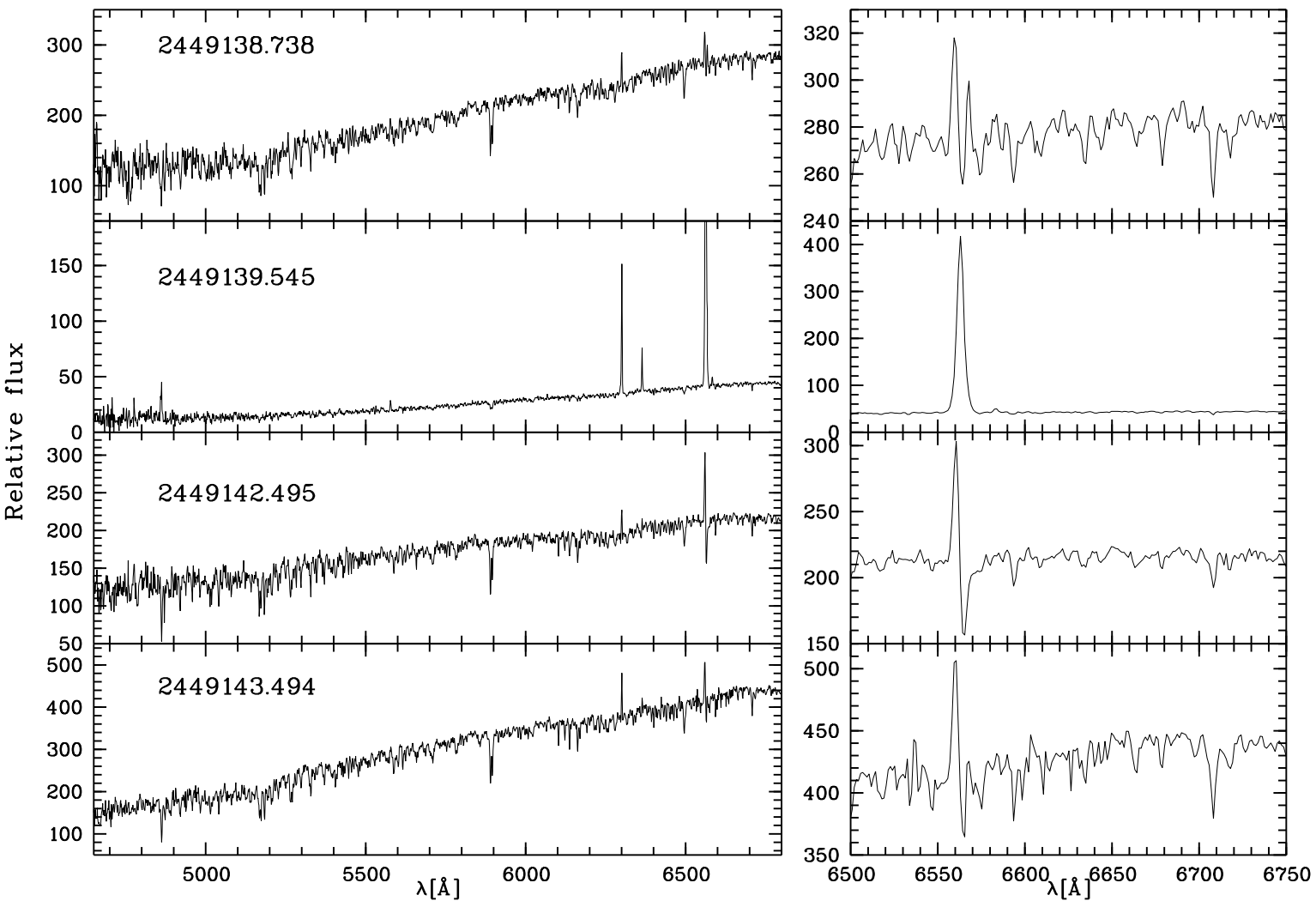

Fig. 26. Atlas of low-resolution spectra of T Cha, continue.
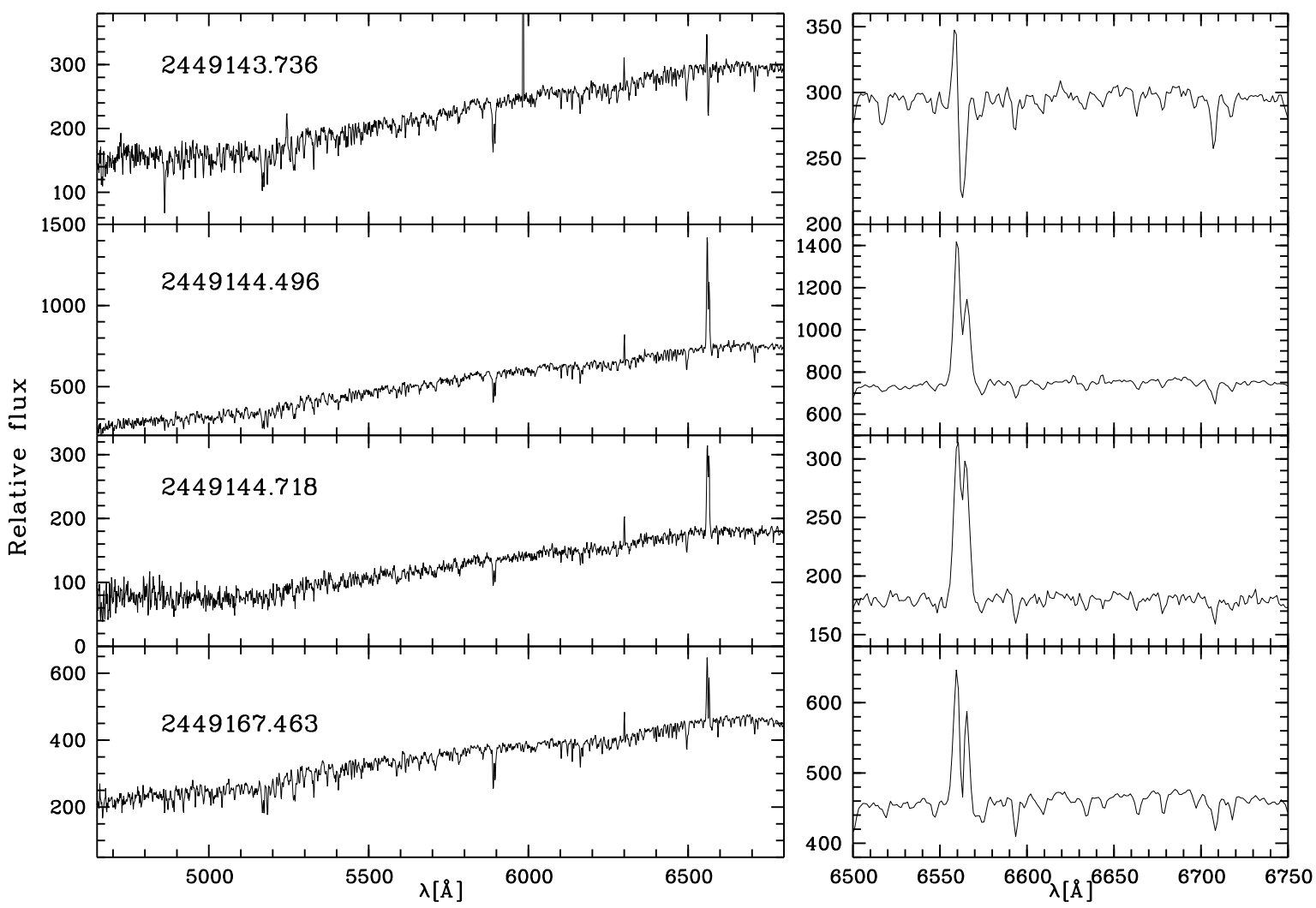

Fig. 27. Atlas of low-resolution spectra of T Cha, continue. 
E. Schisano et al.: Variability of T Cha, Online Material $p 8$
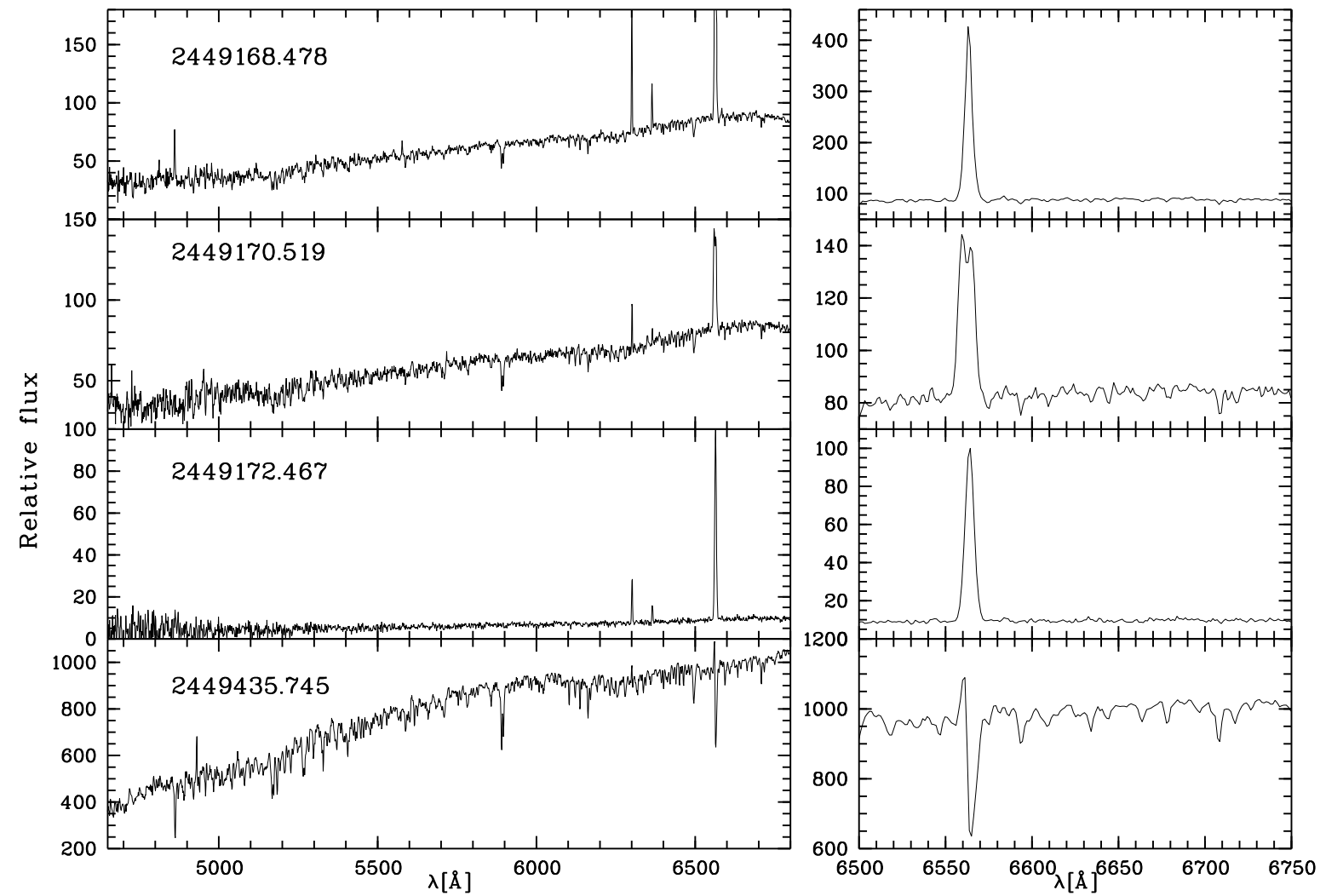

Fig. 28. Atlas of low-resolution spectra of T Cha, continue.
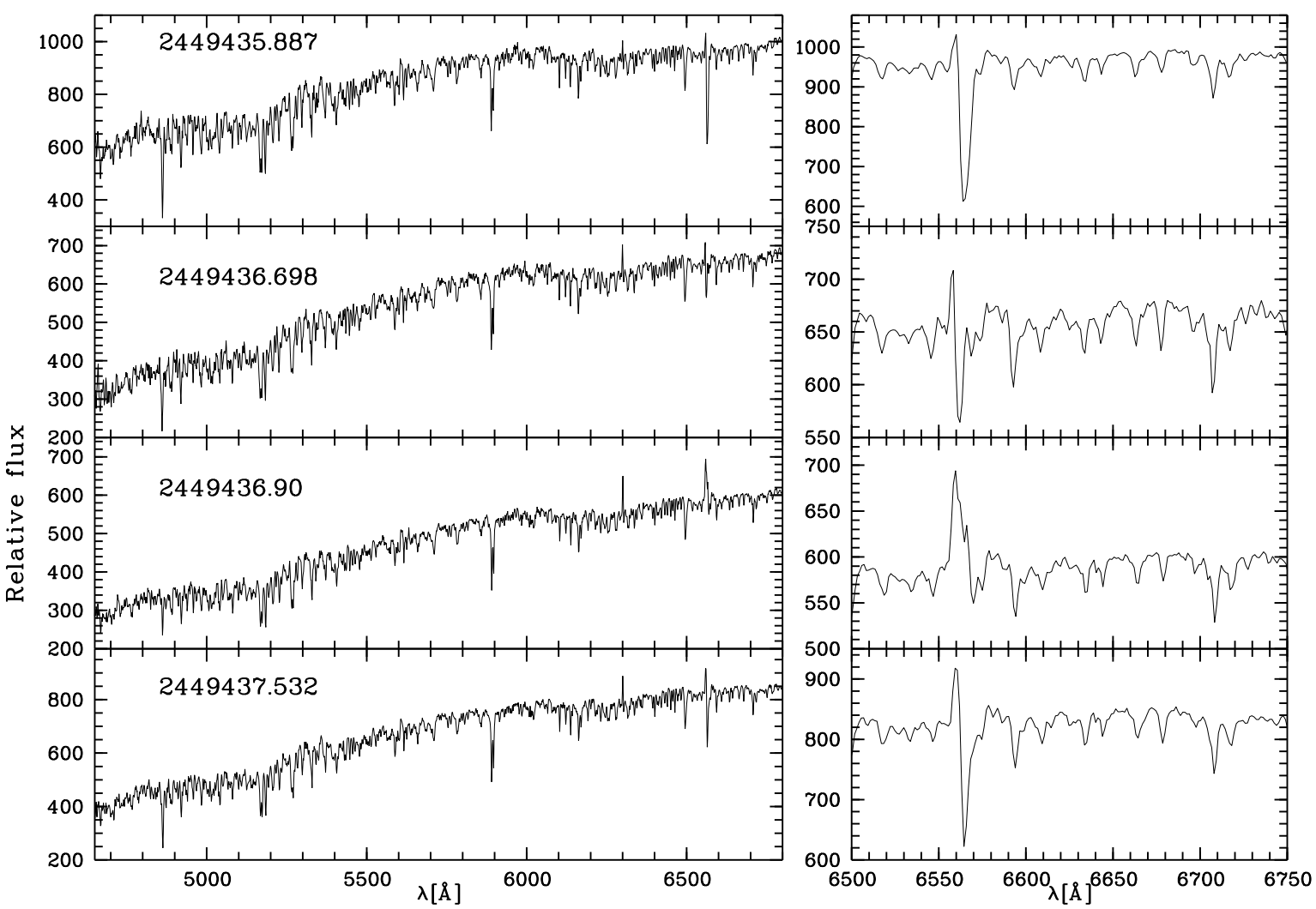

Fig. 29. Atlas of low-resolution spectra of T Cha, continue. 
E. Schisano et al.: Variability of T Cha, Online Material p 9
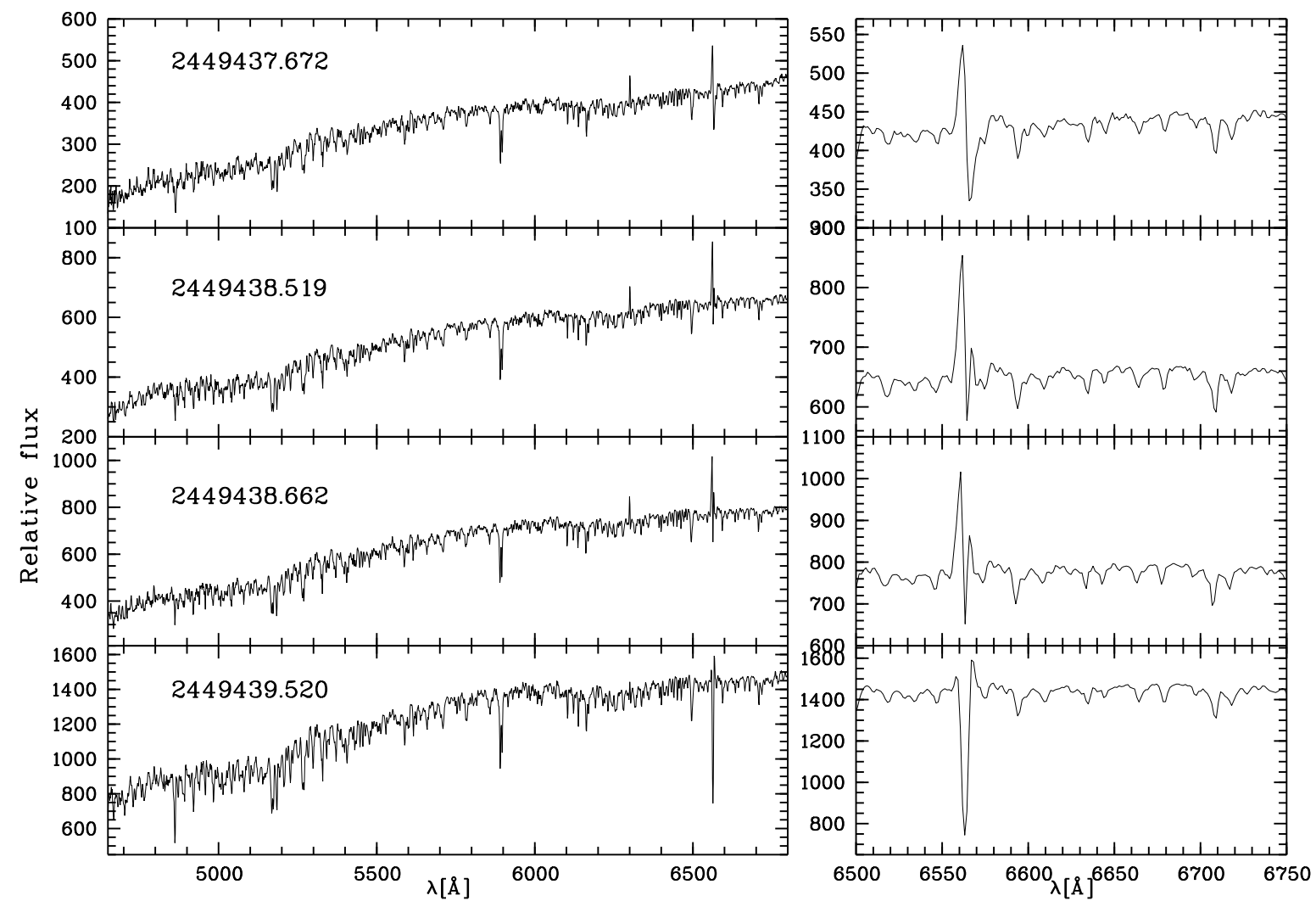

Fig. 30. Atlas of low-resolution spectra of T Cha, continue.
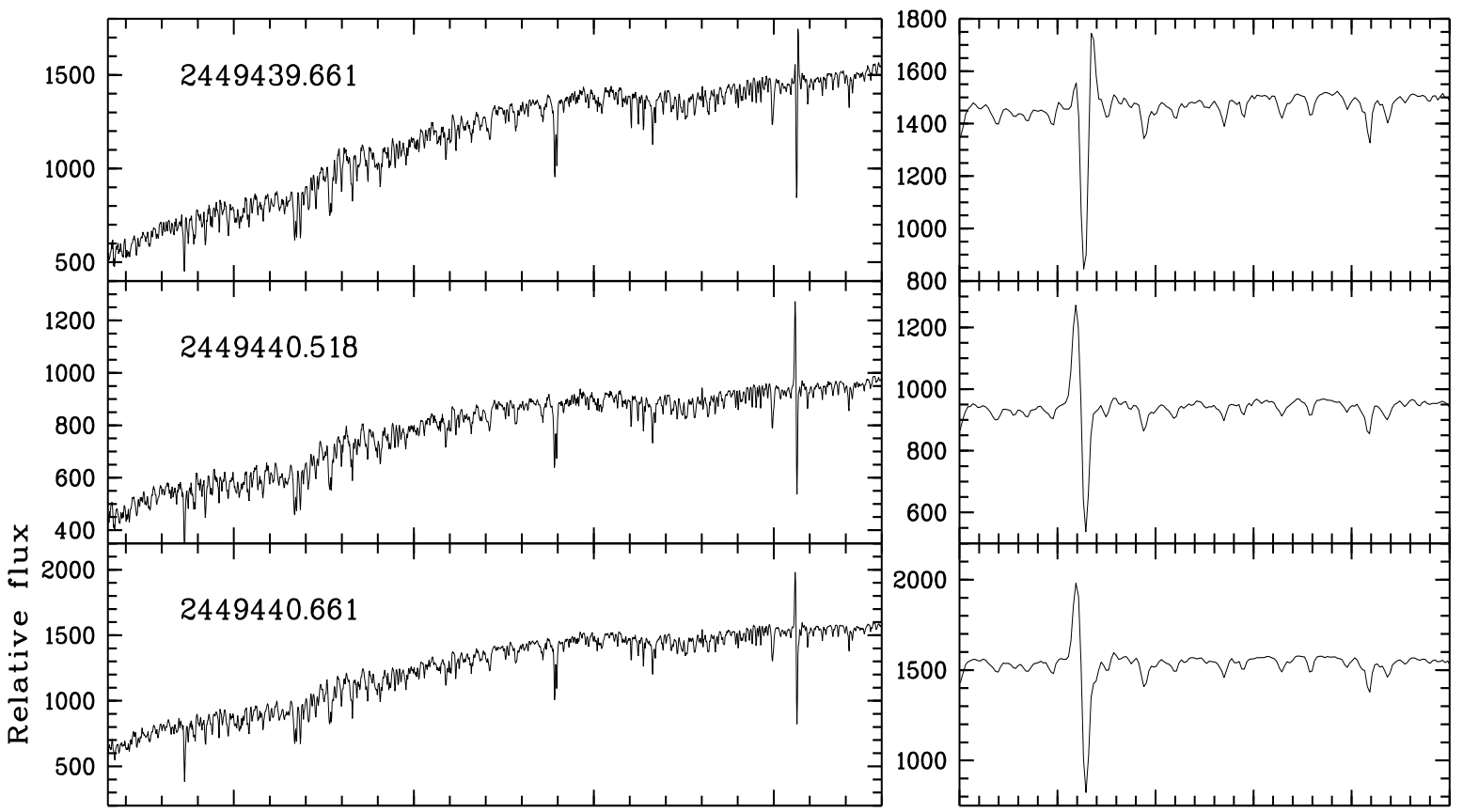

Fig. 31. Atlas of low-resolution spectra of T Cha, continue. 
E. Schisano et al.: Variability of T Cha, Online Material p 10
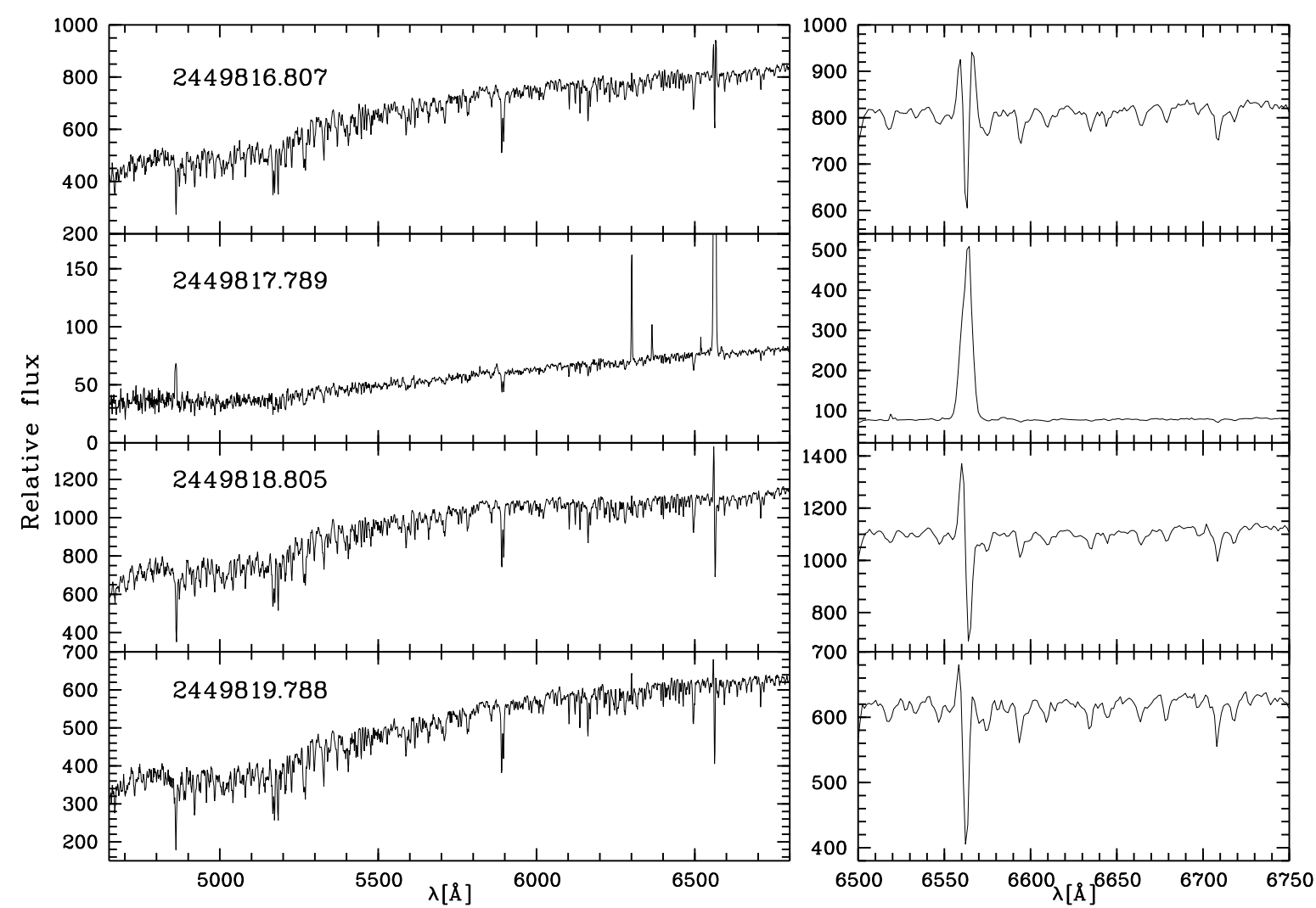

Fig. 32. Atlas of low-resolution spectra of T Cha, continue. 